ARTICLE

\title{
Complete sequences of Schizosaccharomyces pombe subtelomeres reveal multiple patterns of genome variation
}

Yusuke Oizumi (1) ${ }^{1,2}$, Takuto Kaji², Sanki Tashiro ${ }^{2,4}$, Yumiko Takeshita², Yuko Date ${ }^{1}$ \& Junko Kanoh (1) 1,2,3凶

Genome sequences have been determined for many model organisms; however, repetitive regions such as centromeres, telomeres, and subtelomeres have not yet been sequenced completely. Here, we report the complete sequences of subtelomeric homologous (SH) regions of the fission yeast Schizosaccharomyces pombe. We overcame technical difficulties to obtain subtelomeric repetitive sequences by constructing strains that possess single $\mathrm{SH}$ regions of a standard laboratory strain. In addition, some natural isolates of $S$. pombe were analyzed using previous sequencing data. Whole sequences of $\mathrm{SH}$ regions revealed that each $\mathrm{SH}$ region consists of two distinct parts with mosaics of multiple common segments or blocks showing high variation among subtelomeres and strains. Subtelomere regions show relatively high frequency of nucleotide variations among strains compared with the other chromosomal regions. Furthermore, we identified subtelomeric RecQ-type helicase genes, t/h3 and t/h4, which add to the already known $t / h 1$ and $t / h 2$, and found that the $t / h 1-4$ genes show high sequence variation with missense mutations, insertions, and deletions but no severe effects on their RNA expression. Our results indicate that $\mathrm{SH}$ sequences are highly polymorphic and hot spots for genome variation. These features of subtelomeres may have contributed to genome diversity and, conversely, various diseases.

\footnotetext{
${ }^{1}$ Department of Life Sciences, Graduate School of Arts and Sciences, The University of Tokyo, 3-8-1 Komaba, Meguro-ku, Tokyo 153-8902, Japan. ${ }^{2}$ Institute for Protein Research, Osaka University, 3-2 Yamadaoka, Suita, Osaka 565-0871, Japan. ${ }^{3}$ Department of Biological Sciences, Graduate School of Science, The University of Tokyo, 7-3-1 Hongo, Bunkyo-ku, Tokyo 113-0033, Japan. ${ }^{4}$ Present address: Institute of Molecular Biology, University of Oregon, 1370 Franklin Blvd, Eugene, OR, USA. ${ }^{凶}$ email: jkanoh@bio.c.u-tokyo.ac.jp
} 
G enomic DNA sequences provide fundamental information for biological study. The genomes of model organisms, such as Saccharomyces cerevisiae (S. cerevisiae) ${ }^{1}$, Schizosaccharomyces pombe (S. pombe $)^{2}$, Caenorhabditis elegans $^{3}$, Drosophila melanogaster ${ }^{4}$, Arabidopsis thaliana ${ }^{5}$, and Homo sapiens $s^{6-8}$, have been sequenced in the past two decades, and most parts of these sequences have been reported. However, sequencing of long repetitive regions has not been completed because of technical difficulties in sequencing and chromosome allocation of such regions, as well as frequent occurrence of mutations and structural changes caused by chromosome rearrangements, such as recombination, translocation, chromosome breakage, and fusion ${ }^{9-13}$.

Incomplete genomic DNA information can lead to inaccurate data in some experiments. For instance, we are unable to determine the precise chromatin localization of proteins in repetitive regions without actual DNA sequences. Evaluation of protein localization by chromatin immunoprecipitation assays involves PCR with sets of representative primers that target repetitive sequences or Southern blot analysis with representative probes. Chromatin localization values obtained using representative primers or probes merely show averages of all regions that have the target sequences, and they do not reflect actual patterns of chromatin association. Next-generation sequencers (NGSs) do not solve this problem if complete genome sequences are not provided. In addition, there may be uncharacterized genes in unsequenced regions. Therefore, complete sequences of genomic DNA are crucial for accurate analyses and a deeper understanding of model organisms.

Telomeres, which exist at chromosome ends and possess species-specific tandem repeat sequences, play crucial roles in several cellular activities required for cell survival, including protection of chromosome ends, length regulation of telomerespecific repeat DNA, and regulation of chromosome movements during mitosis and meiosis ${ }^{4-17}$. Subtelomeres, which are adjacent to telomeres, have sequences distinct from telomere repeats and generally contain multiple species-specific segments that share high similarity with other subtelomeres. In the budding yeast $S$. cerevisiae, the subtelomeres contain $\mathrm{X}$ and $\mathrm{Y}^{\prime}$ elements, the latter of which includes the open reading frame (ORF) of a helicase gene ${ }^{18}$. In humans, the subtelomeres are mosaics of $\sim 50$ types of common segments containing various ORFs, such as those for the DUX4 gene, which is related to facioscapulohumeral muscular dystrophy, and for the olfactory receptor family genes $9,19,20$. Although substantial knowledge of telomeres has accumulated, research on subtelomeres has progressed slowly compared with research on other chromosomal regions because of technical difficulties caused by long and repetitive nature of this region and partially unknown sequences.

The fission yeast $S$. pombe is one of the most commonly used yeast model organisms for biological study. It preferentially proliferates as haploid in nutrient-rich media and possesses only three chromosomes (chromosome 1 [Ch1], 5.6 Mb; Ch2, 4.6 Mb; $\mathrm{Ch} 3,3.5 \mathrm{Mb}$ ), which enables the whole package of genetic analyses, such as screening for both dominant and recessive mutations, and generation of cells with circular chromosomes by deleting telomere DNA. S. pombe subtelomeres spanning $\sim 100 \mathrm{~kb}$ are subdivided into two regions of $\sim 50 \mathrm{~kb}$ each, the telomereadjacent and telomere-distal regions (Fig. 1a). The telomereadjacent regions ( $\mathrm{SH}$ [subtelomeric homologous] regions) of subtelomeres contain $S \bar{H}$ sequences, which share high similarity $\left(>90 \%\right.$ identity) with at least one other subtelomere in S. pombe $e^{21}$ and form heterochromatin structures ${ }^{22,23}$. This $S H$ region is subdivided into two regions, the telomere-proximal SH-P and telomere-distal $S H-D$ regions by their different features in 972 strains (Fig. 1a; see below). In contrast, the $S H$-adjacent regions
(SU [subtelomeric unique] regions) share almost no sequence similarity with other subtelomeres, but exhibit common highly condensed chromatin structures called knobs ${ }^{24}$ (Fig. 1a). Because of high sequence similarity, it is very difficult to distinguish individual $\mathrm{SH}$ regions of subtelomeres at different chromosome ends and to assemble repetitive subtelomeric sequences accurately, even if we use latest NGSs. Therefore, parts of $S H$ regions remain un-sequenced for 19 years after vast majority of the $S$. pombe genome sequence was reported (S. pombe genome database, PomBase: http://www.PomBase.org/status/sequencingstatus) $)^{2}$ (Fig. 1b). Previously, parts of the four $S H$ regions have been cloned and sequenced (pNSU series, see below) ${ }^{25}$; however, they have not yet been allocated to specific subtelomeres (see PomBase).

\section{Results}

Construction of strains containing single $\mathrm{SH}$ regions of 972 . To overcome the difficulty in allocating each $\mathrm{SH}$ sequence to a specific subtelomere, we constructed strains containing single $\mathrm{SH}$ regions of the standard S. pombe strain $972\left(h^{-}\right)$. Strain 972 used in this study (a derivative of the original 972 (ref. ${ }^{26}$ )), which has not been crossed with other strains, possesses four $\mathrm{SH}$ sequences (SH1L, SH1R, SH2L, and SH2R, as shown in Fig. 2a) adjacent to the telomeres of $\mathrm{Ch} 1$ and $\mathrm{Ch} 2$, but no SH sequence in $\mathrm{Ch} 3$ (note that some descendent strains of 972 possess a partial $(\sim 16 \mathrm{~kb}$ long) $\mathrm{SH}$ sequence adjacent to the telomeres of the left and/or right arms of Ch3; see Supplementary Fig. 1) 21,27,28. We previously produced the $S D 5$ ( $\underline{S} H$ deletion 5) strain, in which all five

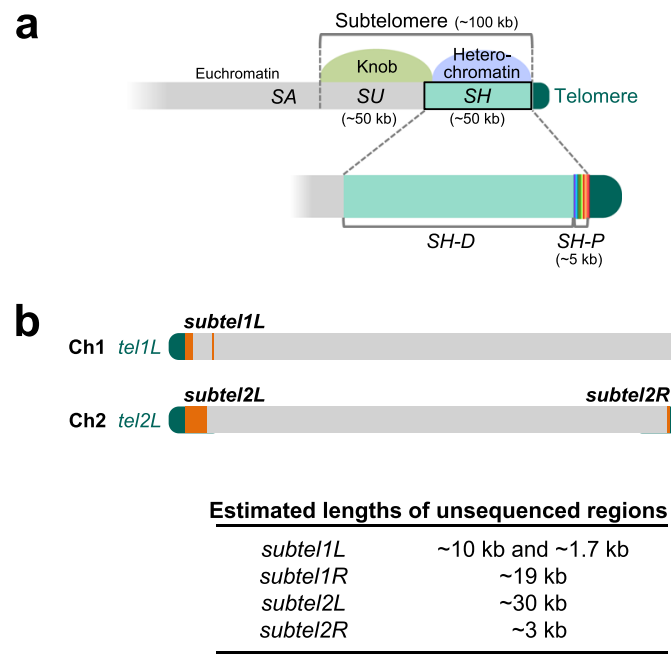

Fig. 1 Structures and previously unsequenced regions of subtelomeres in S. pombe 972 strain. a Schematic illustration of the structures of subtelomeres $(\sim 100 \mathrm{~kb})$ of $\mathrm{Ch} 1$ and $\mathrm{Ch} 2$ in strain 972 . The $\mathrm{SH}$ region $(\sim 50 \mathrm{~kb})$ shows high sequence identity (>90\%) with other subtelomeres. Subtelomeric heterochromatin is formed around the $\mathrm{SH}$ region ${ }^{21}$. An SH sequence is composed of two characteristic regions, $S H-P(\sim 5 \mathrm{~kb})$ and $S H-D$. In contrast, the $\mathrm{SH}$-adjacent $\mathrm{SU}$ region ( $5 \mathrm{O} \mathrm{kb}$ ) shows low sequence identities with other subtelomeres, but forms a highly condensed knob structure that is shared among them ${ }^{24}$. SA indicates a subtelomereadjacent euchromatin region. $\mathbf{b}$ Schematic illustration of unsequenced regions of subtelomeres in $\mathrm{Ch} 1$ and $\mathrm{Ch} 2$ of strain 972 according to PomBase (indicated by orange boxes). Tel1L, tel/R, tel2L, and tel2R indicate telomeres at the left and right arms of $\mathrm{Ch} 1$ and those of $\mathrm{Ch} 2$, respectively. Subtel1L, subtel $1 R$, subtel $2 L$, and subte $2 R$ indicate subtelomeres at the left and right arms of $\mathrm{Ch} 1$ and those of $\mathrm{Ch} 2$, respectively. Lengths of unsequenced regions are estimated based on the assumption that these $\mathrm{SH}$ sequences show high similarity with that of subtel2R of PomBase. Note that $\mathrm{Ch} 3$ is omitted in this panel (see Fig. 2a for the ends of Ch3 in strain 972). 
a 972

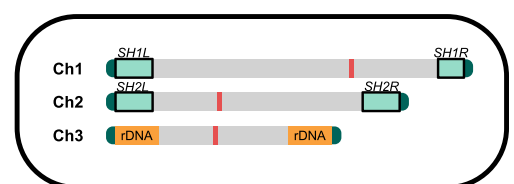

SD5

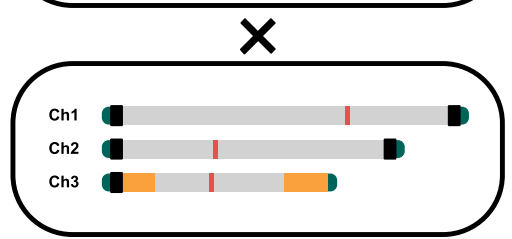

b

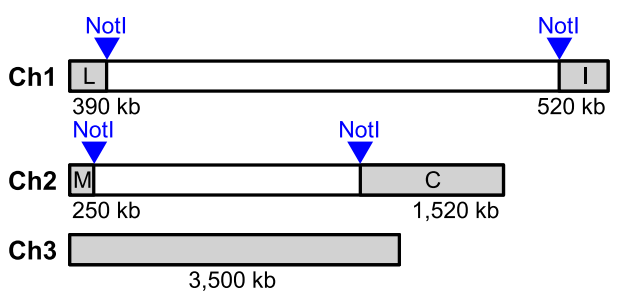

d

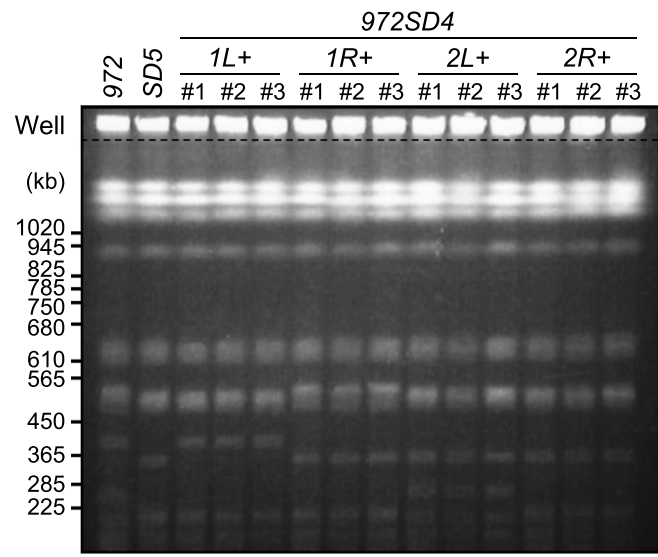

$\mathrm{EtBr}$

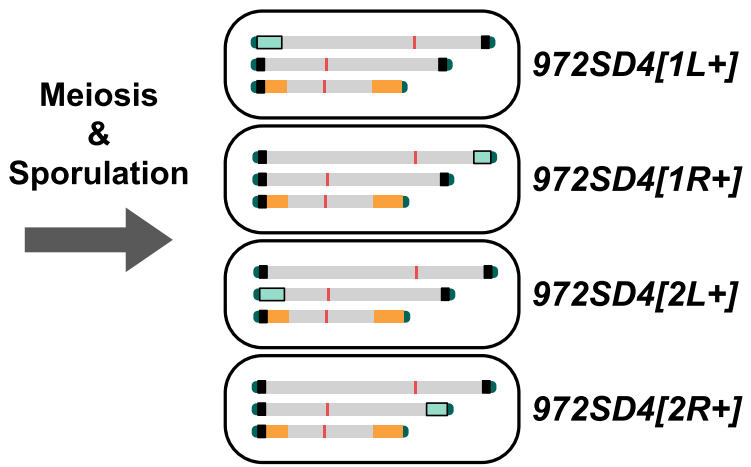

C

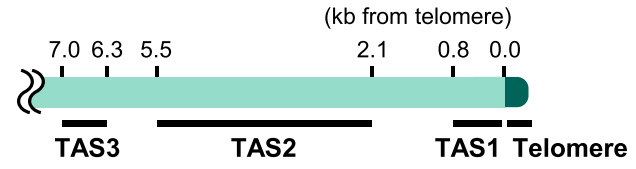

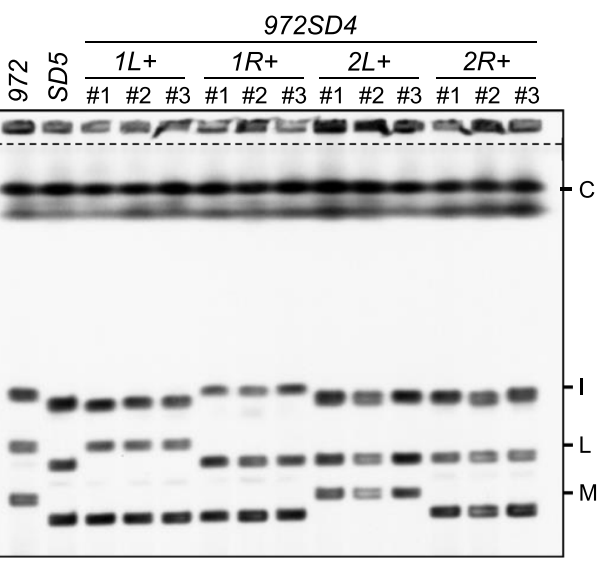

Telomere

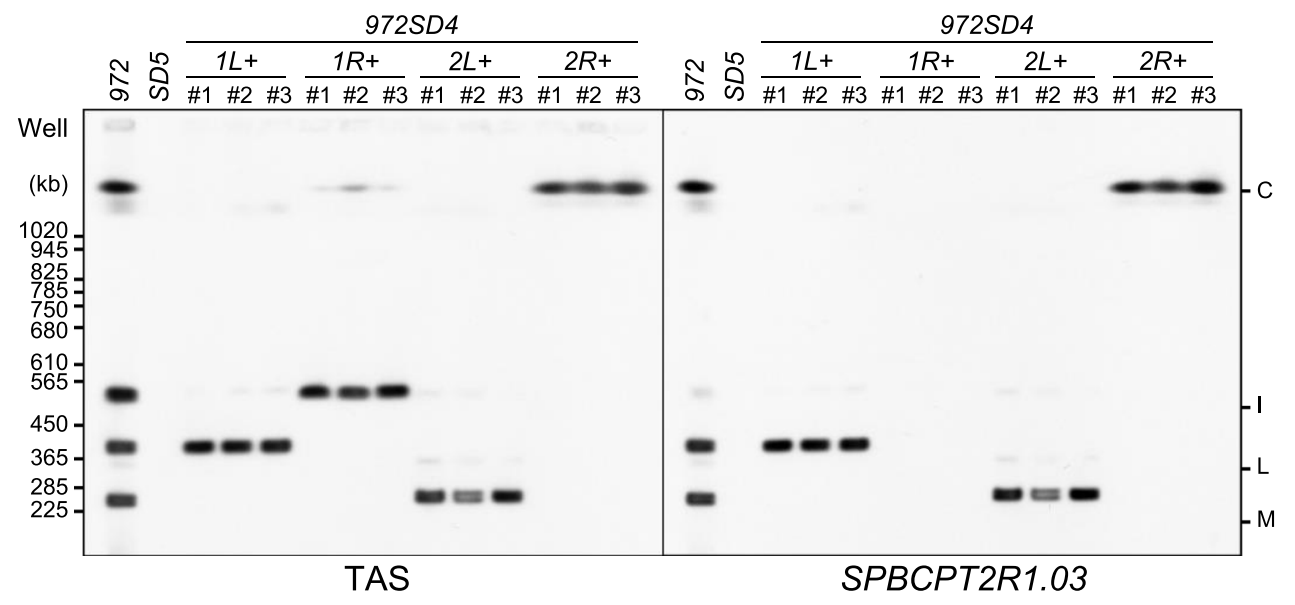

$\mathrm{SH}$ regions that are located at both ends of $\mathrm{Ch} 1$ and $\mathrm{Ch} 2$, and the left end of $\mathrm{Ch} 3$ were replaced with a marker gene (his ${ }^{+}$or $\mathrm{ura}^{+}$) in a nonstandard strain JP1225 background ${ }^{21}$. Strain 972 was crossed with $S D 5$, and the first or second filial progeny were analyzed for the presence or absence of each $S H$ region by pulse- field gel electrophoresis (PFGE) followed by Southern blotting (Fig. 2a-d; also see "Methods" section). We screened for strains that exhibit a single band of telomere-associated sequence $(\mathrm{TAS})^{29}$ (i.e., $\mathrm{SH}$ ). We obtained strains that contain a single $\mathrm{SH}$ region of 972 and named them $972 S D 4[1 L+, 1 R+, 2 L+$, or $2 R+]$, 
Fig. 2 Construction of 972SD4 strains containing a single SH region of strain 972. a Construction strategy of 972SD4 strains. Pale green boxes, SH regions adjacent to telomeres; black boxes, marker genes ( $h i s 7^{+}$or $\mathrm{ura}^{+}{ }^{+}$) that replaced $\mathrm{SH}$ regions ${ }^{21}$; orange boxes, rDNA repeats; and red boxes, centromeres. Strains 972 and SD5 were crossed, and meiosis and sporulation (spore formation) were induced. Progeny with single SH regions were obtained. b Schematic illustration of telomere-containing Notl restriction fragments (shown in gray). Fragments L, I, M, and C contain SH1L, SH1R, SH2L, and $S H 2 R$, respectively. The size of each fragment is shown underneath. c Schematic illustration of the positions of DNA fragments detected by probes for telomeres and TAS (TAS1-3). Shown are the distances from telomeres in pNSU70. d Analyses of the chromosome end structures in 972 SD4 strains. Notldigested chromosomal DNAs of three independent strains of each 972SD4 were analyzed by PFGE followed by Southern blotting using telomere, TAS, or SPBCPT2R1.03 ORF probes. EtBr, ethidium bromide staining of the gel after PFGE. Note that SH1R lacks part of the SH region homologous to SPBCPT2R1.03 according to PomBase. Consistently, 972SD4[1R+] strains did not show an SPBCPT2R1.03. signal (also see Fig. 5). These data were reproduced twice.

since they carry deletions of four $\mathrm{SH}$ regions in the original SD5 and one intact $\mathrm{SH}$ region from 972. Each intact $\mathrm{SH}$ region in 972SD4 was named as 972SD4-SH1L, 972SD4-SH1R, 972SD4SH2L, or 972SD4-SH2R.

Cloning and sequencing of $\mathrm{SH}-\mathrm{P}$ regions. From the data in PomBase, multiple common segments aligned in a mosaic pattern were expected for the $S H-P$ region (see below). In order to accurately assemble such repetitive sequences, we amplified the $S H-P$ region $(\sim 5 \mathrm{~kb})$ in each $972 S D 4$ by $\mathrm{PCR}$ and cloned into a vector. Partial deletion series of the $S H-P$ fragments was constructed by digesting the plasmids with restriction enzymes followed by treatment with exo- and endo-nucleases. Re-circularized plasmids that carry the $S H$ - $P$ fragments with various lengths were sequenced using primers that anneal to the vector (Supplementary Fig. 2, see "Methods" section).

SH-P regions exhibit highly variable mosaic structures consisting of common segments. We classified the sequences of $\mathrm{SH}$ $P$ regions of the $972 S D 4$ strains, a part of $S H 2 R$ in PomBase (PomBase-SH2R), and insertions in the pNSU series in PomBase $^{25}$ into common segments (A-X) as follows. First, the sequences of $\mathrm{SH}-\mathrm{P}$ regions were classified into common segments that meet the criteria of $\geq 50 \mathrm{bp}$ and $>95 \%$ identity using NCBI nucleotide BLAST (blastn) program (v2.10.0+, https://blast.ncbi. nlm.nih.gov/Blast.cgi). Then, gaps between the segments were classified into additional segments that meet the criteria $\geq 14$ bp and $>95 \%$ identity. Segments were classified into variants (e.g., A1 and A2) that meet the criteria of $100 \%$ identity (Fig. 3a and Supplementary Fig. 3). To reduce the number of common segments, exceptional rules were applied for subtypes $\mathrm{C}(\mathrm{C} 1-3)$ and $\mathrm{C}^{\prime}, \mathrm{E}, \mathrm{E}^{\prime}$, and $\mathrm{E}^{\prime \prime}, \mathrm{K}$ and $\mathrm{K}^{\prime}$, and $\mathrm{S}$ and $\mathrm{S}^{\prime}$, which contain different copies of several common sequence motifs that show $100 \%$ identities except for motifs in subtype C, c1-8 (Fig. $3 \mathrm{~b}$ and Supplementary Fig. 3). SH-P regions in two independent strains $(\# 1$ and \#2) of $972 S D 4[1 L+], 972 S D 4[1 R+]$, and $972 S D 4[2 L+]$ exhibit $100 \%$ sequence identity, suggesting that no mutation or rearrangement have been introduced to the $S H-P$ sequences of these 972SD4 strains during crossing, amplification by PCR, cloning using Escherichia coli (E. coli), and construction of serial deletion mutants. In contrast, two strains of $972 S D 4[2 R+]$ contain different variants of segment D, D1 and D3, which show differences in two nucleotides, suggesting that the two point mutations at segment $\mathrm{D}$ and/or interchromosomal recombination have occurred in 972 or $972 S D 4[2 R+]$ (Fig. 3a and Supplementary Fig. 3d).

We found that none of the SH-P regions of 972SD4 show the same pattern in the alignment of segments to each other; however, two pairs of regions, 972SD4-SH1L and pNSU21/65, and 972SD4-SH2L and pNSU64, each exhibit the same segment patterns over the whole $S H-P$ regions, suggesting the possibility that these pairs were derived from the same subtelomeres. However, the compositions of the $\mathrm{C}$ and $\mathrm{D}$ variants are different
(C1 vs. $\mathrm{C} 2$ or $\mathrm{C} 3$, and $\mathrm{D} 1$ vs. D3; Fig. 3a, gray dotted lines). We found that segments $\mathrm{C}$ in $972 \mathrm{SD} 4$ strains are particularly different from those of pNSUs (Fig. 3a, gray dotted lines); i.e., variant C1 is the majority in the $S H-P$ sequences of $972 S D 4$, whereas variant $\mathrm{C} 2$ is the majority in those of pNSUs. These data suggest that segment $\mathrm{C}$ is prone to mutation and recombination possibly due to its highly repetitive structure (Fig. $3 \mathrm{~b}$ ).

In contrast to 972SD4-SH1L and 972SD4-SH2L, 972SD4-SH1R and $972 S D 4-S H 2 R$ show combinations of pNSU patterns (Fig. 3a). Moreover, subtypes $\mathrm{E}^{\prime \prime}$ and $\mathrm{K}^{\prime}$, and variant $\mathrm{H} 2$ are unique to 972SD4-SH1R among the SH-P sequences of 972SD4 and PomBase-972, suggesting that multiple times of mutation and recombination have occurred at 972SD4-SH1R. Surprisingly, 972SD4-SH2R exhibits a pattern different from a part of the PomBase-SH2R sequence; indeed, the pattern of PomBase-SH2R is found in a part of $972 S D 4-S H 1 L$, implying that $S H 1 L$ and $S H 2 R$ have exchanged their chromosomal positions over repeated rounds of cell divisions.

Although we identified several variants for each segment, these variants are not randomly combined, and partial sequences show the same alignments; for instance, there are two common alignments: P1-Q1-----V-Q3 (in 972SD4-SH1L, PomBase-SH2R, pNSU21/65, and pNSU70/77) and E-F2----P3-Q3 (in 972SD4SH2L, 972SD4-SH2R \#1, 972SD4-SH2R \#2, and pNSU64). Overall changes in the segment and variant compositions imply that $\mathrm{SH}$ $P$ regions are prone to nucleotide change and chromosome rearrangement.

$S H-P$ regions exhibit high variation in segmental arrangement among 972 strains and natural isolates of $S$. pombe. To elucidate how $\mathrm{SH}-\mathrm{P}$ regions have changed in the course of culturing or evolution, we next extracted uncharacterized sequences of the $\mathrm{SH}$ $P$ regions in various natural isolates of $S$. pombe in J. Bähler's laboratory in UK (JB strains), using previous raw data by Tusso et al. (long-read sequencing using Nanopore MinION and PacBio RS II) ${ }^{30}$ (Fig. 4a, see "Methods" section for details). Our analyses on chromosome configuration suggested that chromosome end fragment of $C h 1 R$ has been swapped with that of $C h 2 R$ in JB934 in comparison with 972, as described previously (Supplementary Fig. 4$)^{30}$. Although long-read sequencing data lack accuracy, we were able to analyze patterns of segments $(\mathrm{A}-\mathrm{X})$ and subtypes, but not at the variant level (see figure legends of Fig. 4 for details).

JB22 (972), another clone of 972, has SH regions only at the ends of Ch1 and Ch2, not in Ch3, as in the strain 972 in our laboratory. However, SH-P regions in JB22 (972) have different segment patterns and lengths from those in 972SD4 except for SH2R, which does not contain a segment alignment homologous with PomBase-SH2R. These data indicate that chromosome rearrangement occurs frequently in $S H-P$ regions during culturing in laboratories (Figs. $3 a$ and $4 b$ ).

Segment patterns and lengths of $S H-P$ regions in each 972 strain are divergent, whereas JB760, JB1174 (except for SH1R), JB858, and JB1197 exhibit almost uniform segment patterns and lengths among the subtelomeres (Fig. $4 \mathrm{c}, \mathrm{d}, \mathrm{f}, \mathrm{h}$ ). It is unclear 


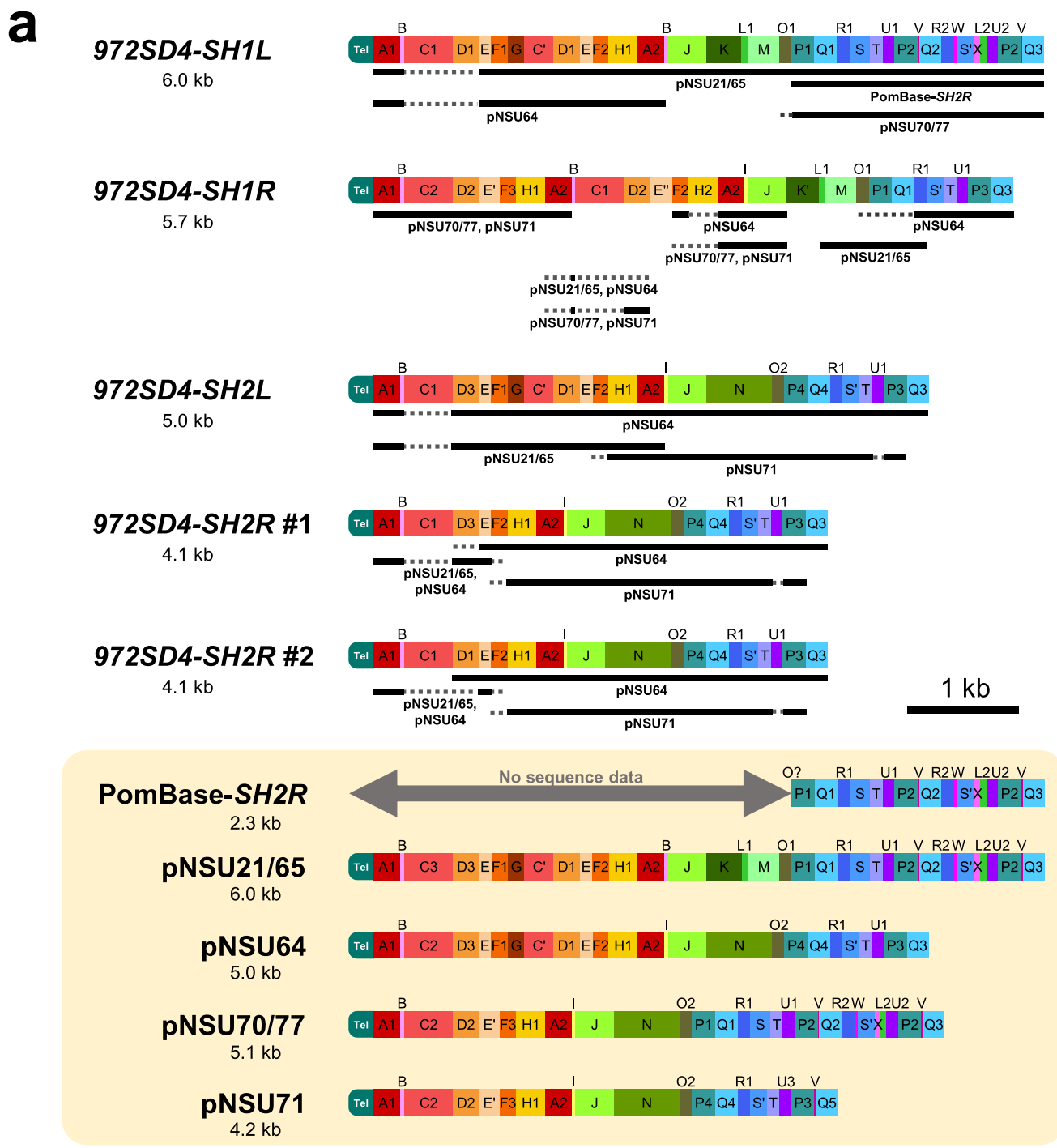

b

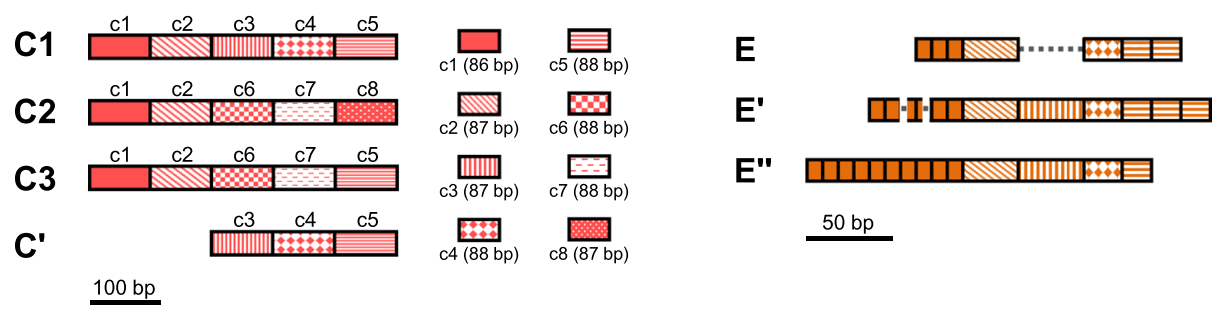

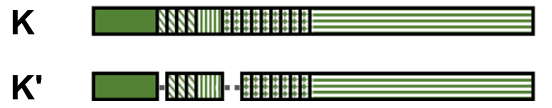

$100 \mathrm{bp}$

which type is the original $S H-P$ in $S$. pombe; however, the divergent property is likely ancestral because repeated interchromosomal rearrangements will result in uniform patterns of $S H-P$ regions.

We detected some sequences that have not been found in 972 strains but shared by several subtelomeres in JB strains

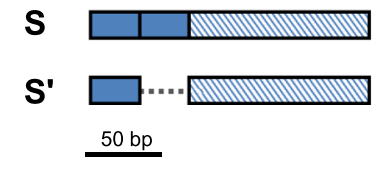

(indicated by black boxes $\alpha-\delta$ in Fig. 4 and Supplementary Fig. 5). Particularly, partial sequences of $S H 1 L$ and $S H 2 R$ in JB873 (segments A-I----- $\Delta \mathrm{P}-\Delta \mathrm{Q}$ containing black boxes) are highly homologous with the $S H-P$ sequences in JB1197 (Fig. $4 \mathrm{~g}$, h). Furthermore, JB934 does not possess any $\mathrm{SH}$ sequence in Ch1L, and JB873 contains partial sequence of long terminal 
Fig. 3 Highly variable SH-P regions consist of common segments in strain 972. a Schematics of SH-P regions. The SH-P sequences were classified into common segments $(A-X)$ and their variants (see Supplementary Fig. 3 for each sequence). SH-P regions of $972 S D 4$ strains, a partial SH2R region in PomBase (PomBase-SH2R), and SH-P regions of a pNSU series in PomBase are shown. The total length of each SH-P region is indicated. Note that $972 S D 4-$ SH1L, 972SD4-SH1R, and 972SD4-SH2L obtained from two independent 972SD4 strains (i.e., \#1 and \#2 clones) are identical, whereas 972SD4-SH2R \#1 and \#2 contain different variants D, D1 and D3. Black bars indicate relatively long regions that show the same segments and variants with those of pNSUs or PomBase-SH2R, whereas gray dotted lines indicate regions that show the same segments but different variants. $\mathbf{b}$ Subtypes of $\mathrm{C}$, E, K, and $\mathrm{S}$ consist of common sequence motifs with different copy numbers. The boxes with the same color and pattern show $100 \%$ sequence identity. Segment $C$ consists of three variants, C1-3, that are composed of homologous sequence motifs, c1-8, that show $>82 \%$ identities with each other (see Supplementary Fig. 3c, $c^{\star}$ for details).

repeat (LTR) of retrotransposon in the $S H-P$ region of $S H 2 L$ (Fig. 4e, g).

Interestingly, there are two major types of structures at chromosome ends, telomere-segment A-segment B (Tel-A-B) and telomere-partial segment F-segment $\mathrm{G}$ (Tel- $\Delta \mathrm{F}-\mathrm{G})$. Tel-A-B is found in 972 and JB760 (Figs. 3a and 4b, c), whereas Tel- $\Delta$ F-G is found in JB1174, JB934, JB858, and JB873 (Fig. 4d-g). We found that the sequences of segments A and B is highly similar to those of partial segment $F$ and segment $G$, suggesting the possibility that homologous recombination (HR) between them resulted in a Tel- $\Delta \mathrm{F}-\mathrm{G}$ structure (Supplementary Fig. 6a). Other possibilities are that nucleotide changes have occurred in either A-B or $\Delta$ F-G (Supplementary Fig. 6b), or that the G-rich sequences located at the telomere-proximal ends of segment $\mathrm{A}$ and $\Delta \mathrm{F}$ formed $\mathrm{G}$-quadruplex-like structures and stalled replication fork progression ${ }^{31}$, which resulted in chromosome breakage accompanied by de novo telomere synthesis (Supplementary Figs. 3 and $6 \mathrm{c}$ ). Overall diversity of the $S H-P$ regions in JB strains further support our hypothesis that $\mathrm{SH}-\mathrm{P}$ regions are hot spots for genome evolution.

SH-D regions in 972 strains are variable and accumulate insertions and deletions. We also determined sequences of $S H-D$ regions in $972 S D 4$ strains by integrating the partial $S H-D$ sequences in PomBase and our newly determined $S H-D$ sequences. The integrated $S H-D$ sequences were classified into common block sequences I-XI (>90\% identities between the sequences of the same blocks), and supplemental homologous box sequences $\Psi$ and $\Omega$ (Fig. 5a and Supplementary Table 1). In contrast to the $S H-P$ regions, the $S H-D$ regions in strain 972 do not show duplications or differences in orders of blocks I-XI. However, we found differences in length; multiple insertions or deletions were identified in these regions (Fig. 5b).

There are three big differences between subtelomeres (Fig. 5b, thick lines; note that part of them has been described in a previous study ${ }^{32}$ ). First, there are $3.7 \mathrm{~kb}$ deletions in SH1R, SH2L, and $S H 2 R$ at position 4,520,423 of PomBase-SH2R. Second, there is a $7.1 \mathrm{~kb}$ deletion in $S H 1 R$ at nucleotides $4,514,836-4,507,710$ of PomBase-SH2R. This deletion was detected in the three independent strains of $972 S D 4[1 R+]$ by PFGE-Southern analysis (Fig. 2d). Third, there is a $1.9 \mathrm{~kb}$ deletion in $S H 1 \mathrm{~L}$ at nucleotides 4,500,309-4,498,438 of PomBase-SH2R.

Intriguingly, boxes $\Psi$ exist at the ends of the $3.7 \mathrm{~kb}$ change (Fig. 5a, b, purple and pink boxes, Supplementary Fig. 7a, b, and Supplementary Table 1). Similarly, boxes $\Omega$ exist at the ends of $7.1 \mathrm{~kb}$ changes (Fig. 5a, b, brown, red, and orange boxes, Supplementary Fig. 7c, d, and Supplementary Table 1). These data imply that the deletions and/or insertions have occurred using these homologous sequences. It is noteworthy that among $\Omega$ sequences that are indicated by different colored boxes, there are multiple insertions and deletions in the region where various repeat sequences are arranged intricately, and the sequence of $\Omega S H 1 L-L$ (indicated by a brown box) shows lowest sequence identity (83-85\%) with other red or orange boxes (Supplementary Fig. $7 \mathrm{c}, \mathrm{d})$.

There are also smaller insertions or deletions in $S H-D$ regions (Fig. 5b, thin dotted lines for changes of 10-40 bp compared with PomBase-SH2R). Many of these are observed in no less than two $\mathrm{SH}-\mathrm{D}$ regions, suggesting that these changes have been copied to other $\mathrm{SH}$ regions by chromosome rearrangement. It is also noteworthy that the newly sequenced $S H 2 R$ in $972 S D 4[2 R+]$ contains an insertion at position 4,529,376 of PomBase-SH2R, indicating that this insertion has been introduced to $S H 2 R$ of strain 972 in laboratories after separated from PomBase-972.

To examine stability of the $S H-D$ regions, their DNA structures in two independent strains of 972SD4[1L+], 972SD4[1R+], $972 S D 4[2 L+]$, and $972 S D 4[2 R+]$ were analyzed by PCR using multiple primer sets (PCR products $\mathrm{i}-\mathrm{ix}$ are indicated by gray arrows in Fig. 5b, top. Note that the fragments i-ix in Fig. 5b roughly correspond to blocks I-IX in Fig. 5a). We found that lengths of the all PCR products matched those predicted from PomBase sequence (Fig. 5c), indicating that the overall DNA structures of $S H-D$ regions are stably maintained between PomBase-972 and 972SD4 strains in contrast to those of SH-P regions. We calculated the full length of each $\mathrm{SH}$ region $(\mathrm{SH}-\mathrm{P}$ and $S H-D)$ : SH1L, $61.9 \mathrm{~kb}$; SH1R, $39.1 \mathrm{~kb}$; SH2L, $59.1 \mathrm{~kb}$; and $S H 2 R, 49.5 \mathrm{~kb}$, with $S H 1 R$ having the shortest $S H$ sequence, although these lengths are likely to change through chromosome rearrangements at $\mathrm{SH}$ regions.

To examine evolutional change of $S H-D$ regions, we next analyzed $S H-D$ regions in JB strains using previous NGS data (long-read sequencing) by Tusso et al..$^{30}$. The $S H-D$ regions in JB22 (972) show clear differences compared with those in PomBase-972 and 972SD4, although the overall pattern of common blocks is very similar (Fig. 6a, b). Block IV and a purple box $(\Psi)$ sequences are present in both SH1L and SH2L in JB22 (972), but not in SH2L in 972SD4. In addition, a brown box sequence $(\Omega)$ resides between blocks $\mathrm{V}$ and $\mathrm{VI}$ in $S H 1 L$ and $S H 2 L$ in JB22 (972), whereas the sequence is replaced by a red box sequence $(\Omega)$ in SH2L in $972 S D 4$. These data suggest the possibility that HR has occurred between the $S H-D$ regions in $S H 1 L$ and $S H 2 L$ in JB22 (972). Thus, the $S H-D$ regions in 972 strains are not highly stable; rather, changeable via recombination.

SH-D regions exhibit striking variations among JB strains. The $S H-D$ regions of the other JB strains show striking variation in numbers and orders of block sequences. Furthermore, various box sequences that are not categorized as $S H-D$ in 972 strains are shared by multiple $S H-D$ regions in JB strains (Fig. 6c-h). It is noteworthy that 972 strains have no $\mathrm{SH}$ sequence in $\mathrm{Ch} 3$, whereas JB760, JB1174, JB858, and JB873 possess $S H-P$ and $S H-D$ sequences in Ch3 (Figs. 4 and 6). Intriguingly, some descendent strains of 972 also possess $\mathrm{SH}$ sequences at either or both ends of Ch3 (Supplementary Fig. 1, KYP33 and JP1225). These data suggest two possibilities; one is that another standard strain 975 $\left(h^{+}\right)$contains $\mathrm{SH}$ sequence(s) in $\mathrm{Ch} 3$, and it has been transferred 
a

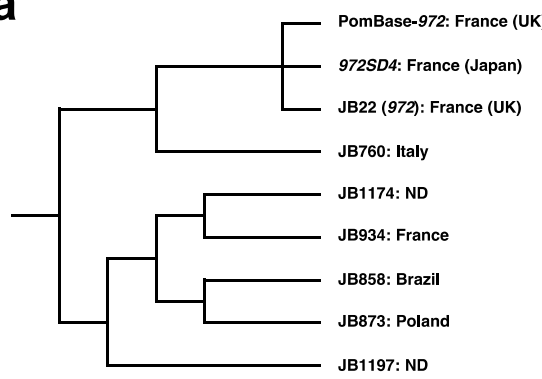

C $\mathrm{JB} 760$

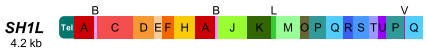

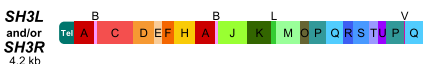

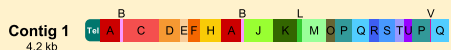

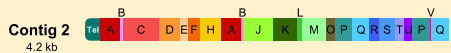

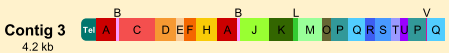

e JB934

Ch1L PomBase-Ch1: 88965-

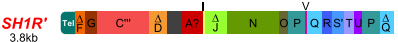

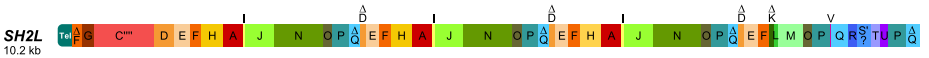

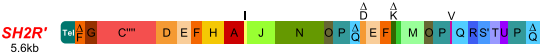

f

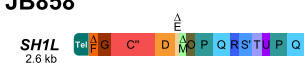

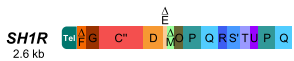

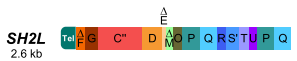

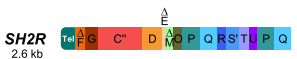

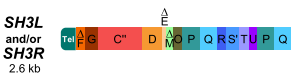

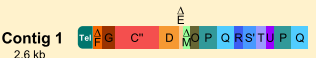

b JB22 (972)

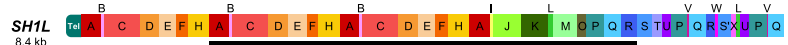

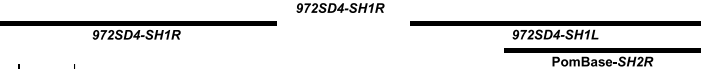

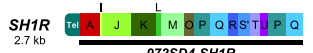

972SDL-SH1L

972504-SH2L 972SD4-SH2R

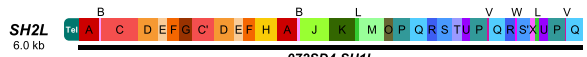

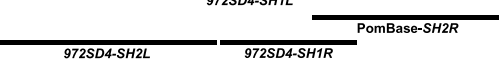

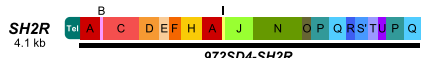

d JB1174

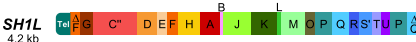

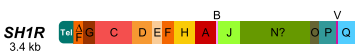

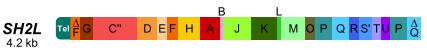

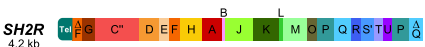

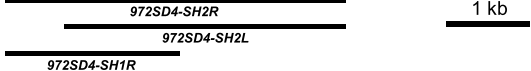

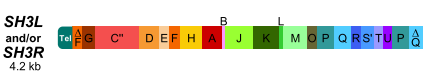


Fig. 4 High variation in SH-P regions in diverse S. pombe strains. a Phylogenetic relationships among 972 and JB strains based on genome-wide sequences according to a previous study by Tusso et al. ${ }^{30}$. Locations where the strains were collected are indicated ${ }^{33}$. ND, not defined. Regarding 972 , locations where the strains are stocked and analyzed are indicated in parentheses. b Schematics of the SH-P sequences in strain JB22 (972) with common segments. Sequences of indicated segments exhibit $90 \%$ or higher identities with those of PomBase or 972SD4. Black bars indicate relatively long regions that show the same segmental arrangements with those of 972SD4 and PomBase-SH2R. c Schematics of the SH-P sequences in strain JB760. Second bar shows an SH-P region of SH3L and/or SH3R of Ch3. Contigs 1-3 include SH-P regions that are not allocated to specific subtelomeres. d Schematics of the SH-P sequences in strain JB1174. $C^{\prime \prime}$ is a subtype of segment $C$ which contains six units of $\mathrm{c}$ motifs (see Figs. $3 \mathrm{~b}$ and $4 \mathrm{i}$ ). $\Delta$ indicates a partial sequence of each segment with no less than 10 bp decrease in total length. "?" indicates 75-90\% sequence identity with the corresponding segment. e Schematics of the $\mathrm{SH}-\mathrm{P}$ sequences in strain JB934. $\mathrm{C}^{\prime \prime \prime}$ and $\mathrm{C}^{\prime \prime \prime \prime}$, subtypes of segment C, contain seven and eight units of c motifs, respectively (see Figs. 3b and 4i). SH1R' and $S H 2 R^{\prime}$ (highlighted in red) indicate that they are located at subtel2R and subtellR in JB934, but overall sequences of telomere-side regions of Ch2R and Ch1R are homologous to those Ch1R and Ch2R in 972, respectively (see Supplementary Fig. 4). Dark gray box in SH1R' indicates a sequence that has not been found in 972 strains and is not shared by the subtelomeres in other JB strains. $\mathbf{f}$ Schematics of the SH-P sequences in strain JB858. Contig 1 includes an $\mathrm{SH}-\mathrm{P}$ region that is not allocated to a specific subtelomere. $\mathbf{g}$ Schematics of the SH-P sequences in strain JB873. Black boxes indicate common segments shared by some of JB strains, although their sequences have not been found in 972 strains (see Supplementary Fig. 5 for their sequences). Note that a partial LTR sequence is inserted at SH2L. $\mathbf{h}$ Schematics of the SH-P sequences in strain JB1197. i Subtypes of segment C. Note that the types of c motifs are not specified due to the relatively low reliability of sequences by long-read DNA sequencing.

JB873, although partial LTR sequences are inserted in block I in JB1174 and JB873 (Fig. 6d, f, g). Intriguingly, the sequence of box $\theta$ is almost identical with that of SAS (spanning $\sim 1.1 \mathrm{~kb}$ ), which was identified as a subtelomere-associated sequence adjacent to block II in Ch3 in the descendent strains of 972 previously ${ }^{21,27}$ (Supplementary Fig. 1b-d). Thus, structures of $S H-D$ regions in Ch3 are highly conserved in $S$. pombe in contrast to other parts of $S H-D$ possibly because the ends of $\mathrm{Ch} 3$ are located in the nucleolus apart from those of $\mathrm{Ch} 1$ and $\mathrm{Ch} 2$ located in the nucleus in vegetatively growing cells ${ }^{17}$, which may restrain interchromosomal recombination between $\mathrm{Ch} 3$ and $\mathrm{Ch} 1$ or $\mathrm{Ch} 2$.

The $S H-D$ regions of JB strains except for JB22 (972) and JB760 showed high variation in their compositions and lengths; however, they share some common features. (1) Blocks I and II are highly conserved. (2) Multiple copies of parts of the $S U$ regions in 972 (indicated by pale blue boxes, $\varepsilon-\eta$, in Fig. 6 a, see Supplementary Table 1 for their positions) are found in the $S H$ regions in these strains (indicated by blue boxes in Fig. 6). (3) Multiple black boxes, $\theta-\tau$, which are not found in 972 are also shared by the subtelomeres of JB strains except for JB22 (972) (Fig. 6). (4) Blocks I and XI (green) and boxes $\varepsilon$ (blue) and $\zeta$ (blue) are found inverted compared with those in 972 (Fig. 6d, e, g). (5) Surprisingly, the $S U$ region of JB858 and the $S H-D$ regions of JB858 and JB873 contain sequences of parts of mitochondrial genome (indicated by Mt in Fig. 6f, g). The overall changes in $\mathrm{SH}$ $D$ regions in JB strains indicate that complexed chromosome rearrangements have occurred in the course of evolution of $S$. pombe even after strain 972 has been isolated.

Subtelomeres are hot spots for mutations. Given that the $S H-P$ regions of 972SD4 contain multiple nucleotide changes compared with those in PomBase-972 (Fig. 3 and Supplementary Fig. 3), we examined whether mutation rates are specifically high in subtelomeres. First, sequences of multiple loci in the $S H-D$ of $S H 2 R$ in two independent 972SD4[2R+] strains (\#1 and \#2) were determined and compared with those of PomBase-972 (Fig. 7 and Supplementary Table 2). We found that regions around the th2 gene locus exhibit high rates of mutations. Most of them are point mutations, but some are changes of numbers of repeat sequences, such as $[\mathrm{T}]_{\mathrm{n}}$. It should be noted that the two $972 S D 4[2 R+]$ strains possess different sequences in the th2 locus ( $99.44 \%$ vs. $99.41 \%$ identities with PomBase). In contrast, the telomere-distal half of $S H-D$ region and all chromosomal regions outside of $S H$, i.e., the $S U$ region, the subtelomere boundary region, the $S A$ region (see Fig. 1a), and various gene loci in Ch1 or Ch2 in the two $972 S D 4$ $[2 R+]$ strains show $100 \%$ sequence identities with those in PomBase-972, indicating strict preservation of their DNA sequences through repeated rounds of cell division. These data suggest that the telomere-proximal half of $S H-D$ regions $(\sim 20 \mathrm{~kb})$, as well as $S H-P$ regions are particularly prone to the accumulation of mutations.

We next examined mutation rates in JB strains using previous NGS data (long-read sequencing by Tusso et al. ${ }^{30}$ and short-read sequencing with high accuracy using Illumina HiSeq 2000 by Jeffares et al. ${ }^{33}$, see "Methods" section for details). Only the chromosomal loci outside of $\mathrm{SH}$ were analyzed because $\mathrm{SH}$ regions are not distinguishable using short reads. We found that JB22 (972) showed 100\% sequence identities with PomBase-972 at the all loci examined (Fig. 7 and Supplementary Table 2), indicating that genome integrity is strictly maintained through repeated rounds of cell division at these loci.

In striking contrast, JB strains other than JB22 (972) exhibit high mutation rates at the $S U$, subtelomere boundary, and $S A$ regions in Ch2R (Fig. 7 and Supplementary Table 2), indicating that subtelomeres are prone to nucleotide changes during the long-time course of $S$. pombe evolution. Interestingly, some JB strains show relatively high mutation rates at the chromosome loci of nonessential genes, especially the rap1 gene (Fig. 7 and Supplementary Table 2). Rap1 is a subunit of the shelterin complex, which protects chromosome ends and regulates various telomere functions ${ }^{15,34}$. Rap1 is recruited to telomeres partly through interaction with a telomere DNA-binding protein Taz1 and associates with multiple proteins to regulate various telomere functions ${ }^{34-36}$. Amino acid changes of Rapl are rarely found in the regions for interactions with its partners (Supplementary Fig. 8). We found one amino acid change, glutamic acid (E) 671 to arginine, in the RCT (Rap1 C-terminal) domain of Rap1, which mediates interaction of Rap1 with Taz1; however, it was suggested that E671 is not involved in their direct interaction ${ }^{37}$. Moreover, one amino acid change, E424 to alanine, is found in the DD (dimerization domain) of Taz1, which is important for Taz1 binding to telomere DNA; however, E424 is located outside of the direct interaction domain ${ }^{38}$. Furthermore, we found that the sequences of telomere repeats in JB strains are highly similar to those in 972 strains. Thus, it seems that the principal functions of Rap1 and Taz1 are conserved during the course of S. pombe evolution. It is likely that the higher mutation rates in the rap1 and tazl genes are because their gene products are more tolerant to amino acid changes than other gene products.

Identification of additional members of the subtelomeric RecQ helicase gene family. In genome sequences in PomBase-972, there are two RecQ helicase genes, $\operatorname{th} 1$ (partial) and th2, which have been allocated to $S H 1 L$ and $S H 2 R$, respectively. Parts of the 
a

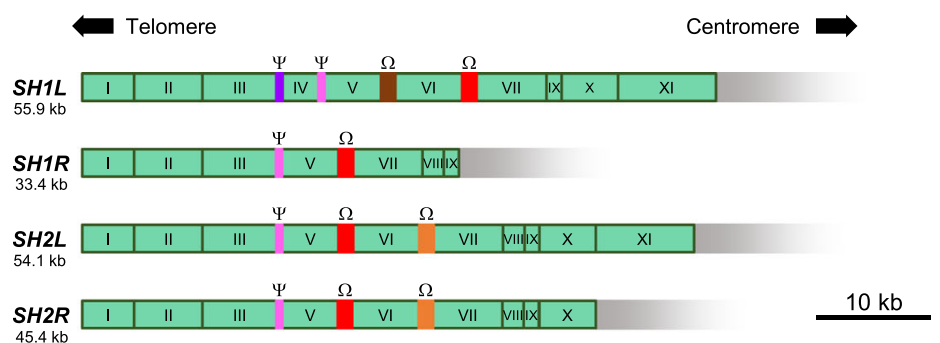

b

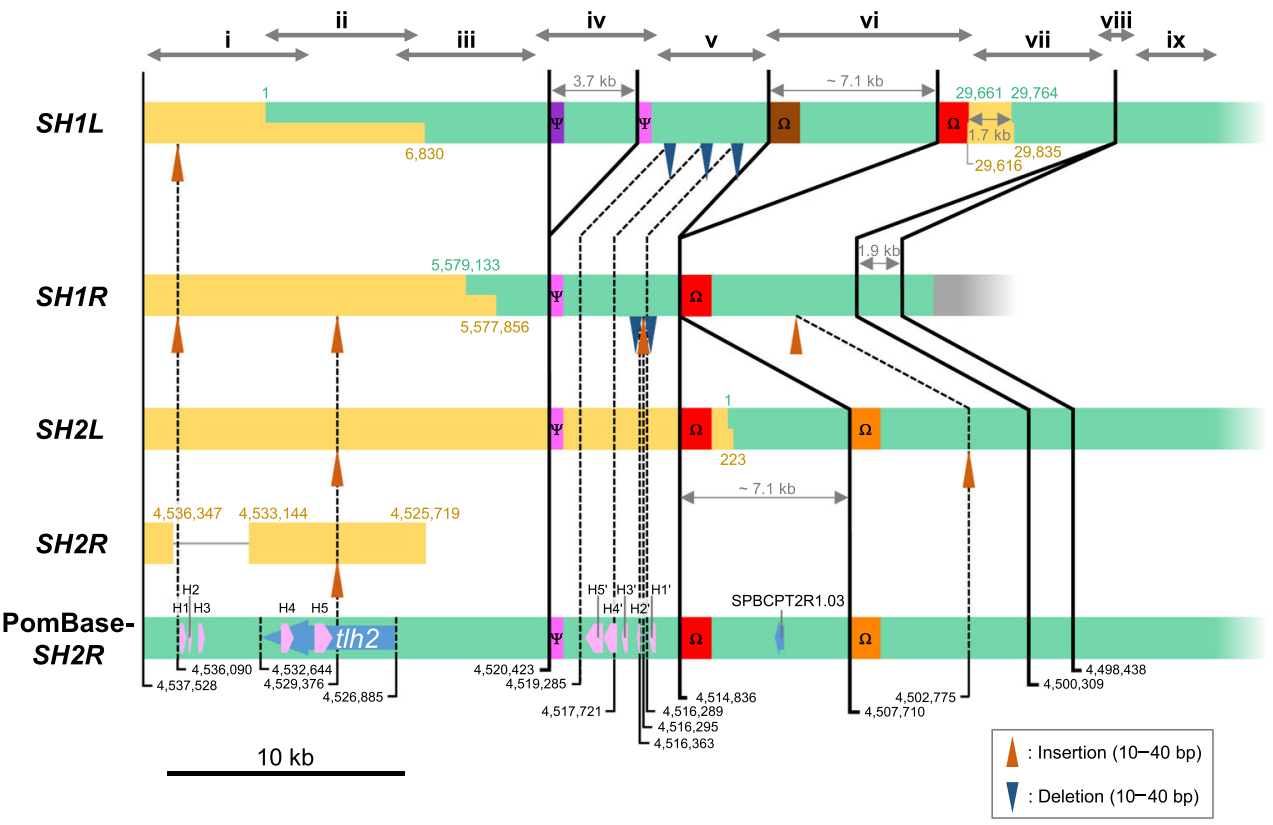

C

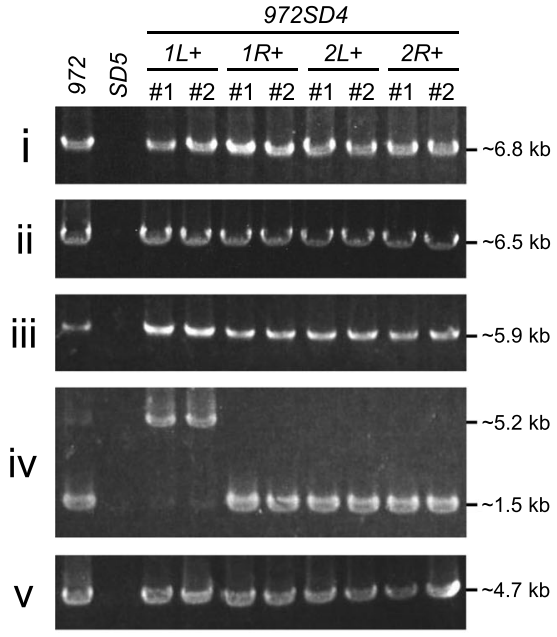

DNA sequences of $t h 1 / 2$ are homologous with the $d h$ repeat sequence of pericentromeres and serve as templates for small interfering RNA (siRNA) produced by RNA interference (RNAi) machinery; further, the siRNA participates in the initiation of subtelomeric heterochromatin formation ${ }^{22,23}$. Our sequencing data of $\mathrm{SH}$ regions newly identified two members of the th gene

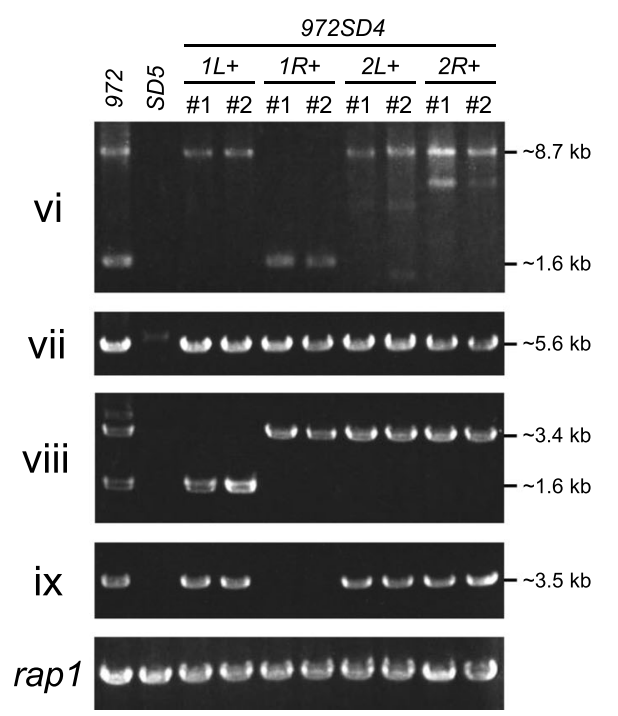

family, th3 and $t h 4$, in $S H 1 R$ and $S H 2 L$, respectively. Thus, genome of strain 972 contains four th genes in total (Fig. 8a and Supplementary Fig. 9).

The descendent strains of 972 possess additional th ORF(s) in Ch3 (Supplementary Fig. 1), that may have transferred from their other chromosome ends or from mating partners. We also found 
Fig. 5 Sequence variations of SH-D regions in 972SD4 and PomBase-972. a Schematic illustration of homologous block (I-XI) and box ( $\Psi$ and $\Omega$ ) sequences in the SH-D regions in 972SD4 and PomBase-972 (see Supplementary Table 1 for their positions in PomBase). All SH sequences are aligned with telomeres to the left and centromeres to the right. $\mathbf{b}$ Comparison of SH-D regions between subtelomeres. Sequences of SH1L, SH1R, and SH2L in PomBase (indicated by green boxes) were combined with sequences newly determined in this study (yellow boxes, showing \#1 clones of each $S H$ sequence), whereas newly sequenced SH2R fragments are shown separately with PomBase-SH2R. Green and yellow numbers indicate positions in each chromosome in PomBase at the ends of overlaps between PomBase and newly determined regions. All SH sequences are aligned with telomeres to the left and centromeres to the right. Insertions (orange arrowheads) or deletions (blue arrowheads) of 10-40 bp are shown in comparison with PomBase-SH2R (thin dotted lines). Insertions and deletions of $<10 \mathrm{bp}$ are omitted in this panel. The thick lines connect the corresponding positions in chromosomes that are boundaries of the long regions with sequence alterations. Purple and pink boxes $(\Psi)$ and brown, red, and orange boxes $(\Omega)$ indicate homologous sequences at the ends of the long sequence changes, 3.7 and $\sim 7.1 \mathrm{~kb}$, respectively (see Supplementary Fig. 7 for the sequences and identities between them). Numbers in black indicate chromosomal position in PomBase-SH2R. Chromosomal positions of insertions and deletions are indicated by the positions in PomBase$S H 2 R$ that are immediately before the changes (closer to the telomeres). Blue arrows in PomBase-SH2R indicate the positions of ORFs for th $2^{+}$and SPBCPT2R1.03. Pale pink arrows in PomBase-SH2R indicate homologous regions ( $\mathrm{H} 1-5$ and their inverted sequences, $\left.\mathrm{H} 1^{\prime}-5^{\prime}\right)$ that are utilized for chromosome fusion when telomeres are lost ${ }^{53}$. Gray arrows at the top indicate ranges of PCR products i-ix analyzed in $\mathbf{c}$. Note that the PCR fragments i-ix in $\mathbf{b}$ roughly correspond to blocks I-IX in a. c Lengths of the SH-D regions in 972 SD4 strains. DNA fragments i-ix were amplified by PCR using genomic DNAs of the 972 (JK107), SD5 (ST3479), and two independent 972SD4 strains (\#1 and \#2) as templates. Approximate DNA lengths estimated by the sequences in PomBase and 972SD4 are indicated on the right. The rap1 locus was amplified as a control. PCRs were performed at least twice.

multiple putative th genes in the $S H-D$ regions of JB strains (Fig. 6, blue arrows). All JB strains possess at least two th genes because th genes are located in block II, which is highly conserved among the strains.

The $t$ lh genes contain multiple nucleotide changes. Examination of the th sequences raised the possibility that the ORF for the th 2 gene is not properly defined in PomBase. In the sequence of PomBase-SH2R, there is an in-frame methionine (Met) codon (uMet [upstream Met]) 543 bases (corresponding to 181 amino acids) upstream of the original first Met codon (dMet [downstream Met]) that was defined by PomBase (Fig. 8b and Supplementary Fig. 9). The sequences surrounding these Met codons match the Kozak consensus sequence (A/GNNATGG, the initiation codon underlined), which participates in the initiation of translation in eukaryotes ${ }^{39}$, suggesting that the uMet may be the true initiation codon for translation.

Unexpectedly, each of the newly sequenced $\operatorname{th} 1-4$ genes of 972SD4 was found to contain multiple nucleotide changes (i.e., point mutations, insertions, and deletions) compared with the th2 gene in PomBase (Fig. $8 \mathrm{~b}$ and Supplementary Fig. 9). There are tandem repeat sequences at the loci where insertion occurred in the long coding region: [ATGACA $]_{n}$ at the most $\mathrm{N}$-terminal insertion (the $6 \mathrm{bp}$ insertion), [CGACAA $]_{\mathrm{n}}$ at the middle insertion (the 18 or $24 \mathrm{bp}$ insertion), and [TGATGG] $\mathrm{n}$ at the most C-terminal insertion (the $6 \mathrm{bp}$ insertion), suggesting that these repetitive sequences are highly prone to recombination (Fig. 8b, orange arrowheads). Because of these changes, the uMet ${ }^{\prime}$ codons of $t$ th $1, t h 2$, and th4 located upstream of uMet are no longer in-frame to the long coding sequence from the dMet codons, and only short coding sequences are predicted because of a termination codon derived by a point mutation (Fig. $8 \mathrm{~b}$, light purple lines with a dark green line). Intriguingly, multiple nucleotide changes, including point mutations, insertions, and deletions, did not introduce premature termination codons within the long ORFs of the th genes in 972SD4. Similarly, the th gene(s) of Ch3 in the 972 descendent strains (KYP33 and JP1225) lack 24 bp immediate downstream of the dMet codons; however, no frame shift occurs, and uMet' codons are supplied by the SAS sequence in the same codon frame (Supplementary Fig. 1d).

RNA expression from the $t$ lh genes in 972SD4. To examine our assumption that the th genes have longer protein-coding sequence than that previously predicted by PomBase, we determined ranges of th RNA expression by reverse transcription (RT)-PCR (Supplementary Fig. 10). We detected RNAs of $t$ th genes that contain at least 950 bases upstream of the dMet codon ( $\sim 400$ bp upstream of the uMet codon). These data suggest that the th genes may have protein-coding sequences that are potentially 543 bases longer than that defined in PomBase (Fig. 8b, a light purple line in the top panel).

To examine whether the th genes in 972SD4 carrying multiple mutations are capable to produce $d h$ RNAs that participate in the formation of subtelomeric heterochromatin, we determined the RNA expression level from the $d h$-homologous region of each $t$ th gene in clr4s cells where the strong gene silencing by subtelomeric heterochromatin is omitted ${ }^{23}$. We found that these four th genes express $d h$ RNAs; however, the expression levels were lower than one-fourth of that in the wild-type strain possibly due to lack of positive feedback regulation by other th RNA expression (Fig. 8c). These results suggest that DNAs of the $t$ th genes are prone to mutations without severely affecting RNA expression.

\section{Discussion}

This study describes in-depth analyses of subtelomeres in $S$. pombe. We obtained complete sequences of subtelomeres in the standard $S$. pombe strain 972 by producing strains with single $S H$ regions. We also extracted $\mathrm{SH}$ sequences of some natural isolates of $S$. pombe strains (JB strains) from previous NGS data. The whole sequences revealed that $S H$ regions are composed of two parts: the telomere-adjacent $S H-P$ region and telomere-distal $S H$ $D$ region. The $S H-P$ region is a mosaic of multiple common segments that vary among subtelomeres and strains, suggesting that this region is highly prone to chromosomal rearrangement during cell divisions. In contrast, the $S H-D$ region shows high sequence similarity among subtelomeres and 972 strains, although there are some insertions, deletions, and chromosomal rearrangement, suggesting that the overall DNA structure of this region is stably maintained during short-term culturing. However, JB strains other than JB22 (972) exhibited striking variation in the structures of $S H-D$ regions, indicating that $S H-D$ regions are also susceptible to chromosomal rearrangement during longterm evolution of $S$. pombe. Interestingly, not only $S H$ but also $S U$ regions exhibit high rates of nucleotide changes among strains, whereas chromosomal regions outside of this region are subject to highly strict genome preservation. Thus, subtelomeres are hot spots for genome evolution and exhibit multiple patterns of genome variation (Fig. 9). 
a PomBase/972SD4

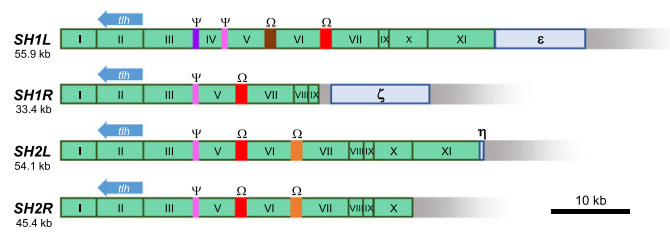

b JB22 (972)

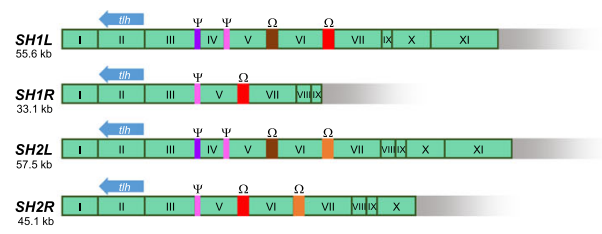

C $\mathrm{JB760}$

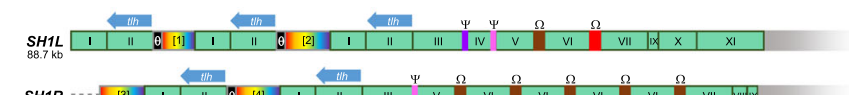

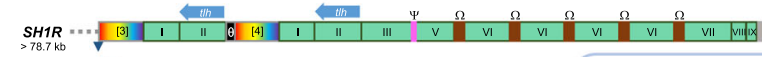

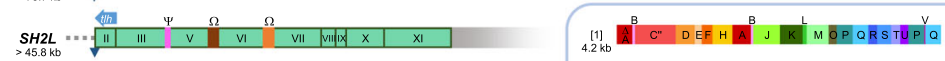

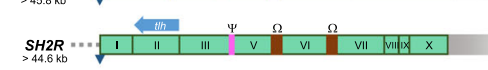

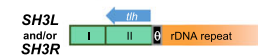

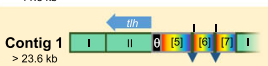

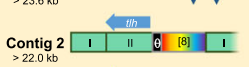

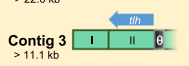

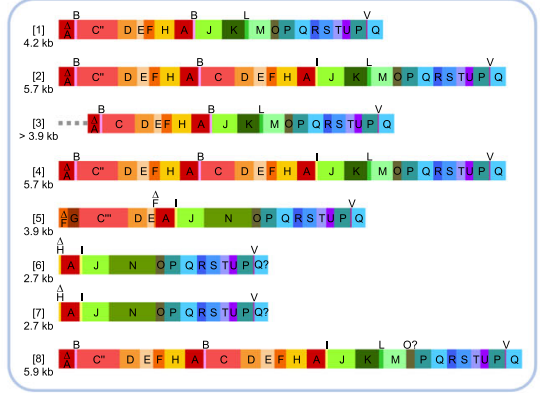

d JB1174

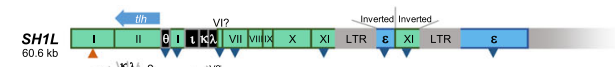

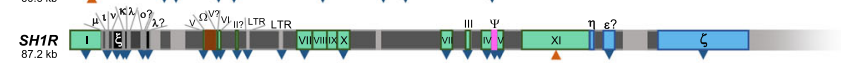

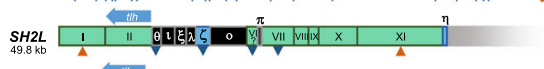

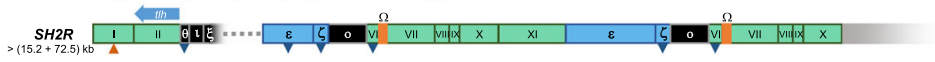

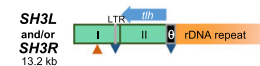

( JB934

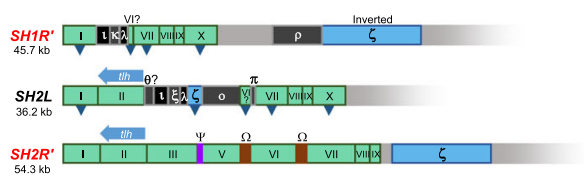

$9 \mathrm{JB873}$

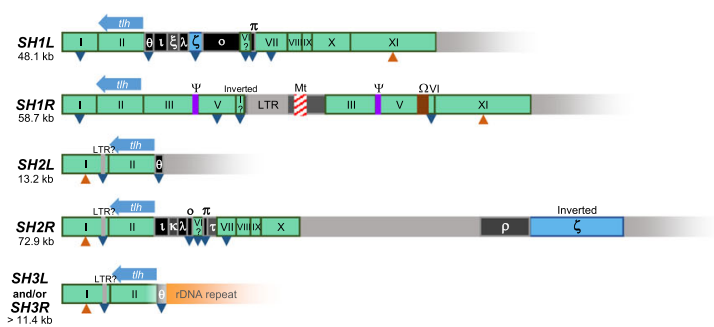

f ${ }_{\mathrm{JB} 858}$

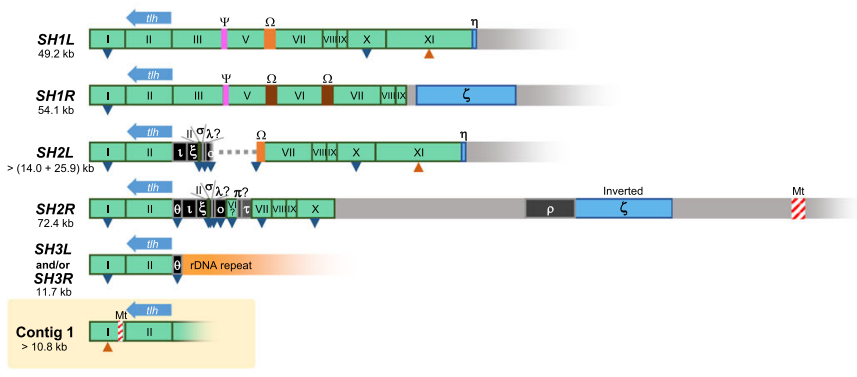

h JB1197

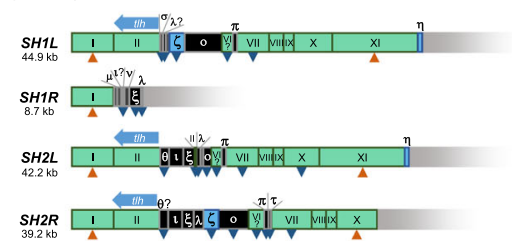

Human subtelomeres ( $\mathrm{SH}$ regions in humans) are also mosaics of multiple common segments that correspond to the SH-P region in $S$. pombe. However, they contain no sequence equivalent to that of the $S H-D$ region, i.e., a relatively long common sequence shared by all subtelomeres ${ }^{9,19}$. Common segments of the same categories are mostly nonidentical ( $\sim 90-100 \%$ identities), and the location and copy number of each segment vary among individuals ${ }^{9,40}$. In S. cerevisiae, the subtelomeres have common $\mathrm{X}$ and $\mathrm{Y}^{\prime}$ elements, and ORFs of proteins such as PAU and FLO families; however, copy numbers of the $\mathrm{Y}^{\prime}$ element and the ORFs are highly variable among strains ${ }^{18,41}$. Based on these findings and studies in other species, along with our results in 
Fig. 6 Sequence variations of SH-D regions in JB strains. a Schematic illustration of homologous block and box sequences in the SH-D regions in $972 S D 4$ and PomBase-972. Note that sequences of pale blue boxes, $\varepsilon-\eta$, in the SU regions are shared by multiple subtelomeres in some of JB strains; and thus, they are defined as SH (see Fig. 6d-h, blue boxes). ORFs of the t/h genes located in block II are indicated by blue arrows. b Schematics of the SH-D sequences in strain JB22 (972) in common blocks and boxes. Sequences of indicated blocks and boxes exhibit at least $90 \%$ identities with those of PomBase-972. Total length of each $S H-D$ region is indicated. c Schematics of the SH-D sequences in strain JB760. Black boxes indicate common sequences that are shared by some other JB strains but not found in 972 strains. Note that the sequence of $\theta$ is homologous with that of SAS (see main text and Supplementary Fig. 1 for the details). Contigs 1-3 are the same as those in Fig. 4c, respectively. Boxes in rainbow color [1]-[8], SH-P-like sequences; "?", sequence with <90\% identity but substantial similarity with the corresponding segment, block or box (E-value $<10^{-10}$ in NCBI nucleotide BLAST [blastn] search); blue triangle, shorter length of the corresponding segment, block or box; gray dotted line, no sequence information available; blocks or boxes with gradation, unreliable sequence information with less than five reads. $\mathbf{d}$ Schematics of the SH-D sequences in strain JB1174. Inverted, opposite sequence direction from that in the corresponding block or box in 972; blue box, sequence shared by multiple subtelomeres as SH in some JB strains; dark gray box, sequence that has not been found in PomBase and not shared by the other JB strains; orange triangle, longer length of the corresponding block or box. e Schematics of the SH-D sequences in strain JB934. f Schematics of the SH-D sequences in strain JB858. Contig 1 is the same as that in Fig. 4f. Shaded box with red lines, an insertion of a part of mitochondrial genome. $\mathbf{g}$ Schematics of the SH-D sequences in strain JB873. $\mathbf{h}$ Schematics of the SH-D sequences in strain JB1197.

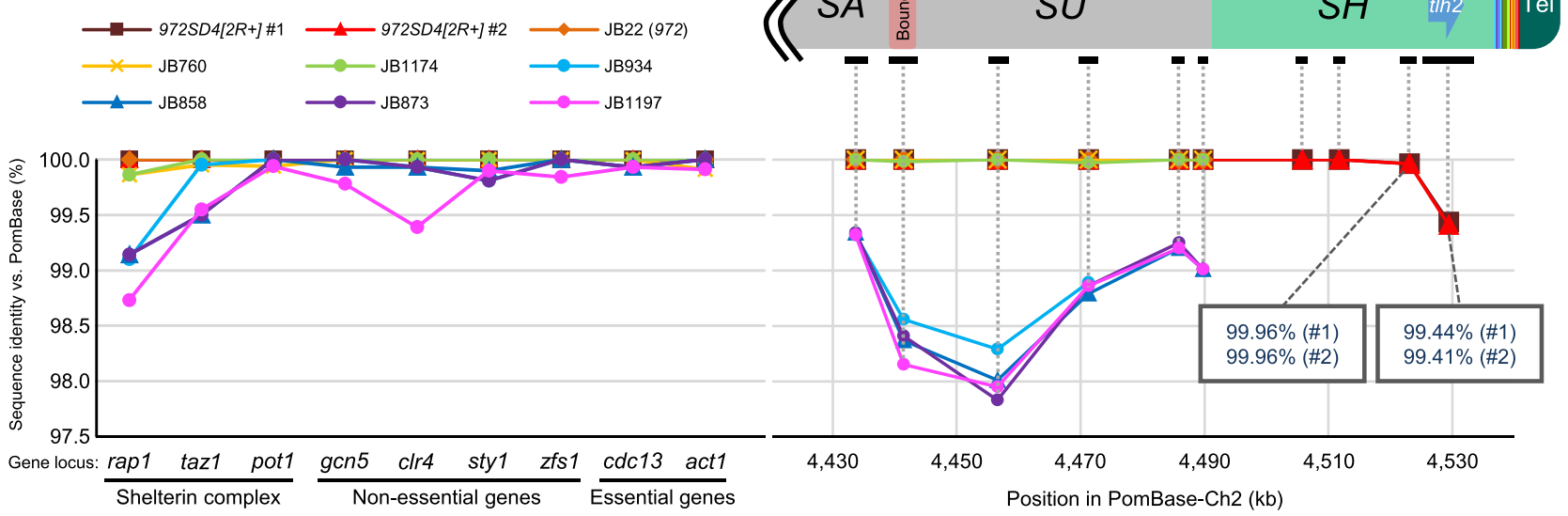

Fig. 7 Subtelomeres are hot spots for sequence variation. Sequence identities between PomBase-972 and other S. pombe strains used in this study. Sequences of the $S H-D$ and SU regions, the subtelomere boundary, and the SA region in Ch2R, and those of other gene loci of three categories were analyzed (see Supplementary Table 2 for the details). Note that two independent 972SD4[2R+] strains (clones \#1 and \#2) contain different sequences around the th/h gene locus.

$S$. pombe, we propose that high variation in $S H$ sequences is a common feature in eukaryotes.

What underlies this high variation of $S H$ regions? First, DNA double-strand breaks (DSBs) are repaired by either HR or nonhomologous end joining. Vegetative cell cycle of the wild-type $S$. pombe strain lacks $\mathrm{G}_{1}$ phase because cells already possess sufficient mass to proceed to $S$ phase when the previous mitosis is completed. Therefore, $\sim 80 \%$ period of the $S$. pombe cell cycle is $\mathrm{G}_{2}$ phase, when HR predominates for DSB repair ${ }^{42}$. Because of the high sequence identities among $\mathrm{SH}$ regions, DNA repair by HR may occur frequently between $\mathrm{SH}$ regions of different chromosomes (interchromosomal repairs), as well as between sister chromosomes (intrachromosomal repairs), which causes gross rearrangement of chromosomes. Second, repetitive sequences within $\mathrm{SH}$ regions may be recognized by HR machineries, causing amplification or deletion of the repeat units. Third, repetitive sequences, including S. pombe telomeres and subtelomeres, are regions intrinsically difficult to replicate during $S$ phase ${ }^{43-45}$. Replication fork collapse and erosion of telomeres and subtelomeres can result in formation of singleended DNA breaks that are repaired by break-induced replication $(\mathrm{BIR})^{46}$. Recent studies suggested that BIR is a highly inaccurate DNA repair mechanism, and causes high levels of mutations and chromosome rearrangements ${ }^{47-52}$. Therefore, BIR may cause high rates of mutations and chromosome rearrangements in $\mathrm{SH}$ regions. Fourth, the $S H-D$ region serve as a fusion point of chromosome circularization when telomeres are $\operatorname{lost}^{53}$. S. pombe has only three chromosomes, which enables cells to survive telomere crisis by selfcircularization of each chromosome ${ }^{29}$. Chromosome end fusions of Ch1 and Ch2 take place between $\mathrm{H} 1-5$ and their inverted sequences, $\mathrm{H}^{\prime}-5^{\prime}$, which are located in blocks I, II, and V (Fig. 5b, pink arrows $)^{53}$. It is possible that chromosome circularization and re-linearization promote chromosome rearrangement of $S H-D$ regions.

Importantly, in the subtelomeres (i.e., the $S H$ and $S U$ regions) of $S$. pombe, there is no gene essential for cell growth under normal culture conditions, and deletion of all $\mathrm{SH}$ regions does not affect cell growth per $\mathrm{se}^{21}$. Genomes of other species also contain multiple copies of the same genes in $\mathrm{SH}$ regions. This may explain why cells can continue to grow, even with mutations in $\mathrm{SH}$ (and $S U$ ) regions, resulting in the accumulation of mutations. The $S U$ regions are known to form knob bodies that are highly condensed chromatin structures, which may prevent precise DNA replication or normal DNA repair, and causes the accumulation of nucleotide alterations ${ }^{21,24}$ (Fig. 9).

Surprisingly, the th genes in 972SD4 contain no nonsense mutation in their ORFs, although they contained various mutations compared with the th2 gene in PomBase. We found that some of the mutations cause alterations in the amino acid sequences of the conserved RecQ motif (Supplementary Fig. 11). Absence of premature termination codon is important for 
a

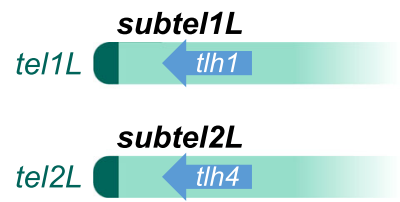

subtel1R

thlh $t e / 1 R$

subtel2R

the tel2R

b
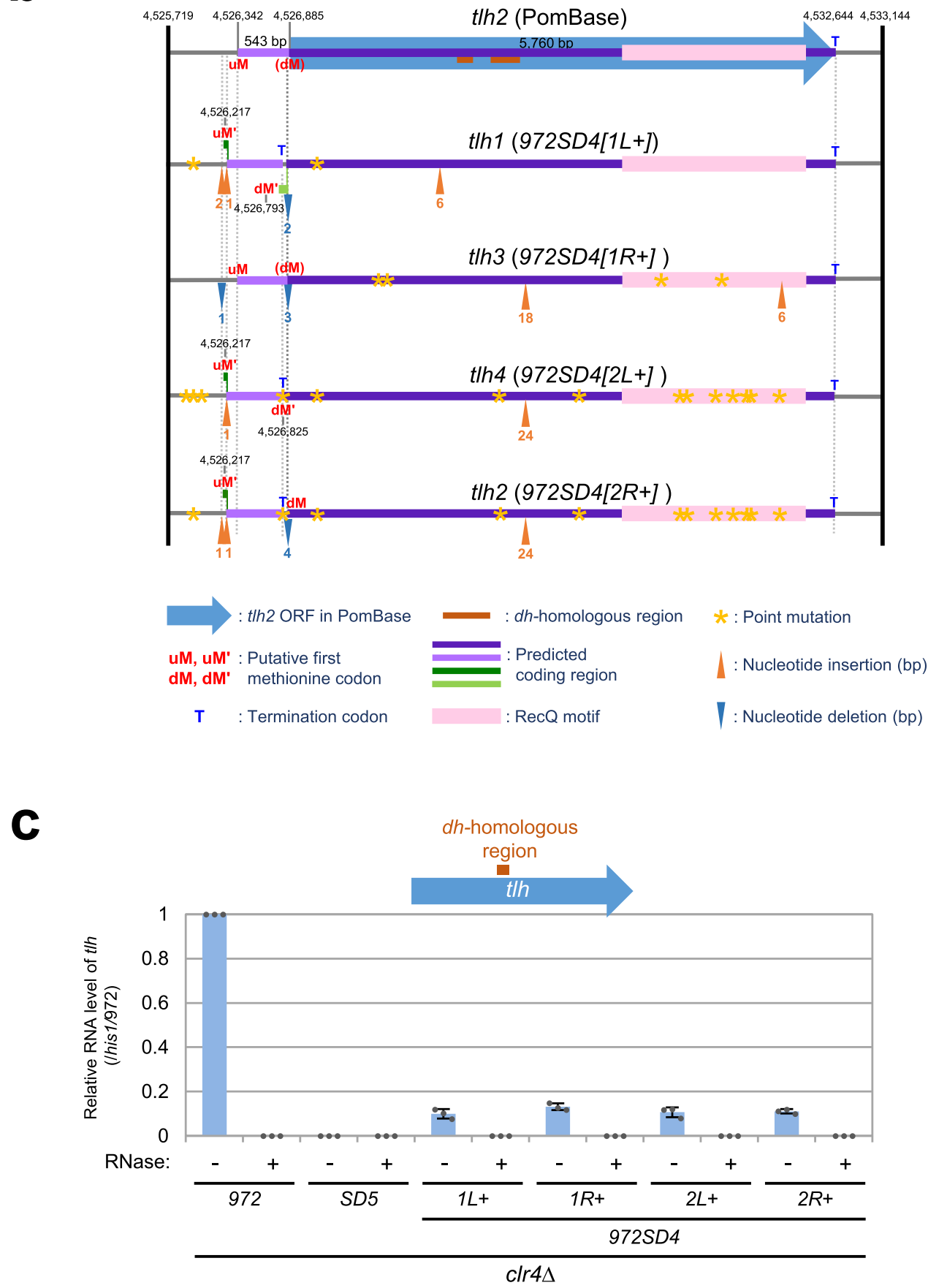

suppression of mRNA degradation mediated by the NMD (nonsense-mediated mRNA decay) mechanism ${ }^{54}$. Thus, it is likely that the principal function of the th genes is to produce RNAs containing $d h$ sequences, and that presence of the th ORFs is advantageous for their normal RNA expression, which induces heterochromatin formation. Although a previous study suggested that the th genes are involved in survival after telomere shortening ${ }^{55}$, functions of the Tlh protein is not clarified yet.

Over 70 years since Dr. Urs Leupold isolated S. pombe standard strains, $968\left(h^{90}\right), 972\left(h^{-}\right)$, and $975\left(h^{+}\right)$, from the Delft culture ${ }^{26}$, it has been believed that these three strains possess almost the same genetic information except for the mating-type genes. 
Fig. 8 Identification of $\mathbf{t} / \mathbf{h}$ genes with multiple mutations. a Identification of $t / h 3$ and $t / h 4$. Chromosomal locations of the four t/h genes are shown. b Summary of predicted ORFs and nucleotide changes in the th genes of 972SD4 compared with the th2 gene in PomBase. The top panel shows the t/h2 locus in PomBase. Seven digit numbers indicate chromosomal positions on Ch2 in PomBase. Blue arrow with a purple line, the t/h2 ORF defined in PomBase; brown bar, $d h$-homologous region; light purple line, predicted coding region which is in-frame with that of the th2 ORF in PomBase; light or dark green line, predicted coding region in the reading frame different from that of the predicted th2 ORF in PomBase; yellow star, point mutation (no frame shift); orange arrowhead, nucleotide insertion (numbers of inserted nucleotides are shown below); blue arrowhead, nucleotide deletion (numbers of deleted nucleotides are shown below); $\mathrm{dM}$ the original first methionine codon of the t/h2 ORF in PomBase, uM putative first methionine codon upstream of $\mathrm{dM}$ for PomBaset/h2, $\mathrm{UM}^{\prime}$ putative first methionine codon for the short ORF, $\mathrm{dM}^{\prime}$ putative first methionine codon for the long ORF, $\mathrm{T}$ termination codon. $\mathbf{c}$ Expression of RNA of the $d h$-homologous region in the t/h genes in strains 972, SD5, and 972SD4 in a clr4 $\Delta$ background was analyzed by quantitative RT-PCR in the presence $(+)$ or absence $(-)$ of RNase. Expression level of $t / h^{+}$relative to that of his ${ }^{+}$was normalized to that in 972 ( $-\mathrm{RNase}$ ). Data are presented as mean values \pm SD (standard deviations). $N=3$ biologically independent experiments (each value is indicated by a gray dot).

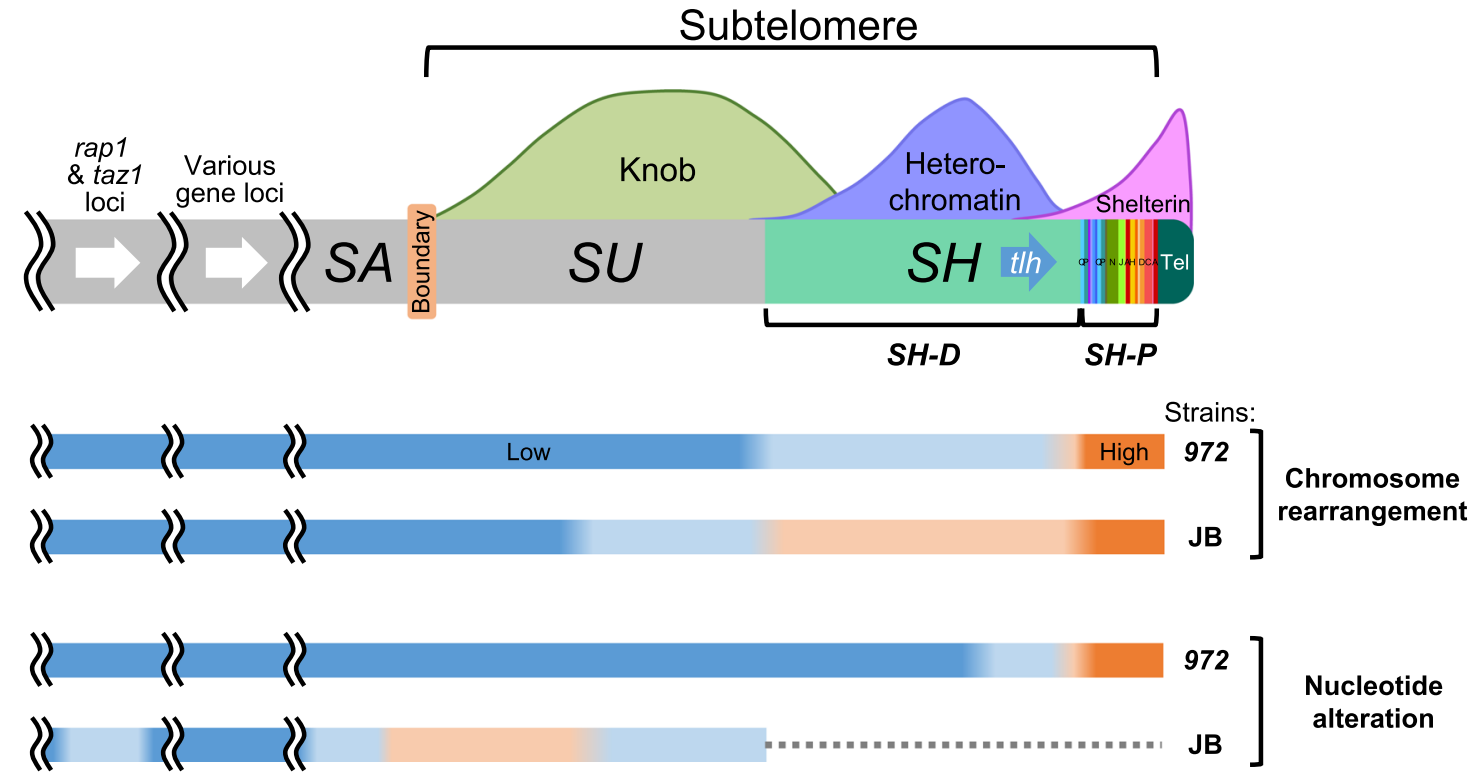

Fig. 9 Summary of features of $\mathbf{S}$. pombe subtelomeres. The S. pombe SH region is composed of two parts: the SH-P region with a mosaic of multiple segments, and the $\mathrm{SH}-\mathrm{D}$ region with multiple blocks and boxes containing deletions and insertions. Colors of bars indicate relative rates of chromosome rearrangement or nucleotide alteration versus PomBase (orange, high rate; blue, low rate). The SH-P region shows high rates of chromosome rearrangement and nucleotide alteration even between the 972 strains (972SD4 and JB22 vs. PomBase), whereas the SH-D region shows lower rates of these changes compared with those in the SH-P region. The SU, boundary, and SA regions and the rap1 and taz1 loci also exhibit medium levels of these changes in the JB strains that are phylogenetically distant from 972. Thus, chromosomal regions around the subtelomeres (and the rap1 and taz1 loci) are prone to genome diversity. Dotted line indicates a region that was unable to be analyzed with the NGS data.

This and previous studies have shown that 972 has no $\mathrm{SH}$ sequence in $\mathrm{Ch} 3$, whereas some of its descendent strains possess $\mathrm{SH}$ region(s) with SAS in Ch3 $3^{21,27,28}$ (Fig. 6 and Supplementary Fig. 1). It is noteworthy that the SAS sequence is absent from the genome of 972 . The three standard strains have been traveling over the world to produce numerous number of descendent strains through mating and meiosis ${ }^{56}$. Therefore, the assumption is that 968 and/or 975 had already had $\mathrm{SH}$ region(s) in Ch3 when they were first isolated.

Taken together, we propose that subtelomeres are highly polymorphic chromosomal regions and contribute to genome evolution. In this study, we have shown that the DNA sequence of the th2 gene has changed from that of the original strain 972 after repeated rounds of cell division (Fig. 8b). Human $\mathrm{SH}$ regions also contain various genes, such as DUX4 (associated with facioscapulohumeral muscular dystrophy) and olfactory receptor genes 9,19 . Thus, the high rates of polymorphisms in $\mathrm{SH}$ regions may contribute to human diversity and sometimes to disease susceptibilities. It is intriguing to investigate correlation between various human diseases and $\mathrm{SH}$ sequences. Overall, genome rearrangement, deletion, insertion, and mutation can cause changes in ORFs, which may result in diversification of species.

\section{Methods}

Strains and general techniques for S. pombe. S. pombe strains used in this study are listed in Supplementary Table 3. Growth media and basic genetic and biochemical techniques used in this study were described previously ${ }^{57-59}$.

Construction of 972SD4 strains. To construct $972 \mathrm{SD} 4$ strains carrying single $\mathrm{SH}$ regions of the standard wild-type strain 972 , the SD5 strain $(S T 3524)^{21}$, in which al five $\mathrm{SH}$ regions were replaced with selective marker genes $\left(h i s 7^{+}\right.$or $u r a 4^{+}$), was crossed with strain 972, and then the progeny were crossed back with the SD5 strain (ST3479 or ST3524) again. The presence or absence of each SH region in the resulting progeny were examined by PFGE followed by Southern blotting.

PFGE. PFGE of NotI-digested chromosomal DNA was performed using a CHEFDR III Pulsed-Field Electrophoresis System (BioRad) under the following conditions: $1 \%$ SeaKem Gold Agarose (Lonza) in $0.5 \times \mathrm{TBE}$; temperature, $10^{\circ} \mathrm{C}$; initial switch time, $40 \mathrm{~s}$; final switch time, $80 \mathrm{~s}$; run time, $18 \mathrm{~h}$; voltage gradient, $6.8 \mathrm{~V} / \mathrm{cm}$; and angle, $120^{\circ}$.

Southern blotting. NotI-digested chromosomal DNA was separated by PFGE and subjected to Southern blotting. Telomeric DNA and TAS fragments (TAS1, TAS2, and TAS3) were excised from pNSU70 ${ }^{25}$, and used as the telomere and TAS probes, respectively. The SPBCPT2R1.03 ORF was amplified by PCR and used as the probe that specifically recognizes the $S H-D$ regions of subtel $1 L$, subtel2L, and subtel2R, but not subtel1R in 972. These DNA fragments were labeled with digoxigenin (DIG), using a DIG High Prime DNA Labeling and Detection Starter 
Kit II (Roche), and signals were detected according to the manufacturer's instructions.

Cloning and sequencing of SH regions. DNA fragments containing $\mathrm{SH}-\mathrm{P}$ regions $(\sim 5 \mathrm{~kb})$ were amplified by PCR from genomic DNA of each $972 S D 4$ strain using Phusion High-Fidelity DNA polymerase (Thermo Fisher) and the following primers.

\section{jk1861:5' - ACTAGTGGATCCCCCTGTAACCACGTAACCTTGTAACC - $3^{\prime}$}

jk1862:5' - GAATTCCTGCAGCCCGGTTTGAGCATCTGTCAGAGGTAA - 3'

Each DNA fragment was inserted at the Smal site of pBluescript SK(-) (Stratagene) using In-Fusion HD Cloning Kit (Clontech). The resulting plasmids were digested with Kpn1 and Xhol at the multiple cloning site of the vector and treated with exonuclease III (Takara) and mung bean nuclease (Takara) for fixed times to obtain deletion series from the XhoI cutting site ( $5^{\prime}$-protruding end). Both ends of the plasmids were blunted with Klenow fragments (Takara) and ligated with DNA ligase (Takara). The re-circularized plasmids were cloned using $E$. coli, $\mathrm{XL1-Blue}$ (recA1 endA1 gyrA96 thi-1 hsdR17 supE44 relA1 lac $\left[\mathrm{F}^{\prime}\right.$ proAB lacI $\left.\mathrm{q} Z \Delta M 15 \operatorname{Tn} 10\left(\mathrm{Tet}^{\mathrm{r}}\right)\right]$ ), and then sequenced using the following primers that anneal to the vector.

$$
\begin{aligned}
& \text { st13 (M13 primer M3) : } 5^{\prime} \text { - GTAAAACGACGGCCAGT - } 3^{\prime} \\
& \text { st14 (M13 primer RV) }: 5^{\prime}-\text { CAGGAAACAGCTATGAC }-3^{\prime}
\end{aligned}
$$

The DNA sequence of $\mathrm{SH}$ - $\mathrm{P}$ was assembled using the overlapping sequences of serial deletion plasmids (Supplementary Fig. 2). Two independent strains of each 972SD4 were analyzed.

DNA fragments $(1.3-2.9 \mathrm{~kb})$ of the $S H-D$ region were amplified by PCR and sequenced, except for the regions with some repeats that were sequenced using the deletion method described above. Two independent strains of each 972SD4 were analyzed.

DNA sequences were determined using BigDye Terminator v3.1 Cycle Sequencing Kit (Applied Biosystems), Prism 3130xl Genetic Analyzer (Applied Biosystems), and DNA Sequencing Analysis Software v5.4 (Applied Biosystems). In addition, some DNA sequences were determined by Eurofins Genomics Inc.

Analyses of previous NGS data of JB strains. To analyze DNA sequences at subtelomeres and other chromosomal loci in JB strains, we utilized previous raw data from NGS by Tusso et al. (long-read sequencing by Nanopore MinION and PacBio RS II; NCBI Sequence Read Archive, PRJNA527756 [https://www.ncbi.nlm. nih.gov/bioproject/PRJNA527756]) ${ }^{30}$ and those by Jeffares et al. (short-read sequencing by Illumina HiSeq 2000; European Nucleotide Archive, PRJEB2733 [https://www.ebi.ac.uk/ena/browser/view/PRJEB2733]) $)^{33}$

De novo assembly of the long reads (by MinION) were performed as follows. Adaptor and its adjacent $(10-20 \mathrm{bp})$ sequences in raw read data were trimmed using Porechop (v0.2.4, https://github.com/rrwick/Porechop) and fastp (v0.20.1) ${ }^{60}$. The trimmed long reads were assembled by Canu 2.0 (ref. ${ }^{61}$ ), using the NIG supercomputer. Chromosome configurations compared with those in strain 972 were analyzed using MUMmer4 (ref. ${ }^{62}$ ).

To determine sequences at $S H$ regions, we collected long reads that contain telomere repeats and telomere-adjacent segments at their ends using NCBI nucleotide BLAST (blastn). The collected reads were classified into several categories using AliView (v1.26) ${ }^{63}$ and MAFFT (v7.453) ${ }^{64}$, and the sequences of each category were combined into one consensus sequence using Minimap2 (v.2.17-r941) ${ }^{65}$ and Racon (v1.4.13) 66 . Locations of the $S H$ sequences were determined by search for homologous sequences in the chromosome assembly described above. Long-read data (by PacBio RS II) were also used to improve the quality of sequences.

To determine sequences other than $\mathrm{SH}$, we searched target sequences in the de novo assembly described above and polished them by Pilon (v1.23) ${ }^{67}$ using shortread data.

RNA analyses. Total RNA was purified from exponentially growing cells as described previously ${ }^{28}$. For the RT-PCR, complementary DNA was synthesized with random primers using a High-Capacity cDNA Reverse Transcription Kit (Applied Biosystems) and analyzed by conventional PCR (Supplementary Fig. 10) or quantitative PCR using a StepOne Real-Time PCR System (Fig. 8c). Primer sequences are listed in Supplementary Table 4.

Reporting summary. Further information on research design is available in the Nature Research Reporting Summary linked to this article.

\section{Data availability}

DNA sequences of newly sequenced $S H$ regions are available in the DNA Data Bank of Japan (DDBJ) under accession codes LC521649 ( 1.7 kb of the $S H-D$ region [SH1L] in $972 S D 4[1 L+] \# 1), \operatorname{LC} 521650(\sim 1.7 \mathrm{~kb}$ of the $S H-D$ region [SH1L] in $972 S D 4[1 L+] \# 2)$, LC521651 (the SH1L region in $972 S D 4[1 L+] \# 1$ ), LC521652 (the SH1L region in $972 S D 4$
$[1 L+]$ \#2), LC521653 (the SH1R region in 972SD4[1R+] \#1), LC521654 (the SH1R region in $972 S D 4[1 R+] \# 2$ ), LC521655 (the SH2L region in 972SD4[2L+] \#1), LC521656 (the $S H 2 L$ region in $972 S D 4[2 L+] \# 2$ ), LC521657 (the $S H 2 R$ region in $972 S D 4[2 R+] \# 1$ ), and LC521658 (the $S H 2 R$ region in $972 S D 4[2 R+] \# 2$ ). Figures $3,5 \mathrm{a}, \mathrm{b}, 6 \mathrm{a}, 7$, and $8 \mathrm{a}, \mathrm{b}$, Supplementary Figs. 3, 6, 7, 9, and 11, and Supplementary Table 2 are associated with these sequence data. Source data are provided with this paper.

Received: 25 February 2020; Accepted: 3 December 2020; Published online: 27 January 2021

\section{References}

1. Goffeau, A. et al. The yeast genome directory. Nature 387, 5 (1997).

2. Wood, V. et al. The genome sequence of Schizosaccharomyces pombe. Nature 415, 871-880 (2002).

3. C. elegans Sequencing Consortium. Genome sequence of the nematode $C$. elegans: a platform for investigating biology. Science 282, 2012-2018 (1998)

4. Adams, M. D. et al. The genome sequence of Drosophila melanogaster. Science 287, 2185-2195 (2000).

5. The Arabidopsis Genome Initiative. Analysis of the genome sequence of the flowering plant Arabidopsis thaliana. Nature 408, 796-815 (2000).

6. Lander, E. S. et al. Initial sequencing and analysis of the human genome. Nature 409, 860-921 (2001).

7. Venter, J. C. et al. The sequence of the human genome. Science 291, 1304-1351 (2001).

8. International Human Genome Sequencing Consortium. Finishing the euchromatic sequence of the human genome. Nature 431, 931-945 (2004)

9. Linardopoulou, E. V. et al. Human subtelomeres are hot spots of interchromosomal recombination and segmental duplication. Nature 437, 94-100 (2005).

10. Arlt, M. F., Durkin, S. G., Ragland, R. L. \& Glover, T. W. Common fragile sites as targets for chromosome rearrangements. DNA Repair 5, 1126-1135 (2006).

11. Kurosawa, K. \& Ohta, K. Genetic diversification by somatic gene conversion Genes 2, 48-58 (2011).

12. Almeida, H. \& Godinho Ferreira, M. Spontaneous telomere to telomere fusions occur in unperturbed fission yeast cells. Nucleic Acids Res. 41, 3056-3067 (2013)

13. Chen, N. W. G. et al. Common bean subtelomeres are hot spots of recombination and favor resistance gene evolution. Front. Plant Sci. 9, 1185 (2018).

14. Chikashige, Y. et al. Telomere-led premeiotic chromosome movement in fission yeast. Science 264, 270-273 (1994).

15. Miyoshi, T., Kanoh, J., Saito, M. \& Ishikawa, F. Fission yeast Pot1-Tpp1 protects telomeres and regulates telomere length. Science 320, 1341-1344 (2008).

16. de Lange, T. How telomeres solve the end-protection problem. Science 326, 948-952 (2009).

17. Fujita, I. et al. Telomere-nuclear envelope dissociation promoted by Rap1 phosphorylation ensures faithful chromosome segregation. Curr. Biol. 22, 1932-1937 (2012)

18. Louis, E. J. The chromosome ends of Saccharomyces cerevisiae. Yeast 11, 1553-1573 (1995)

19. Stong, N. et al. Subtelomeric CTCF and cohesin binding site organization using improved subtelomere assemblies and a novel annotation pipeline. Genome Res. 24, 1039-1050 (2014).

20. Stadler, G. et al. Telomere position effect regulates DUX4 in human facioscapulohumeral muscular dystrophy. Nat. Struct. Mol. Biol. 20, 671-678 (2013).

21. Tashiro, S., Nishihara, Y., Kugou, K., Ohta, K. \& Kanoh, J. Subtelomeres constitute a safeguard for gene expression and chromosome homeostasis. Nucleic Acids Res. 45, 10333-10349 (2017).

22. Cam, H. P. et al. Comprehensive analysis of heterochromatin- and RNAimediated epigenetic control of the fission yeast genome. Nat. Genet. 37, 809-819 (2005)

23. Kanoh, J., Sadaie, M., Urano, T. \& Ishikawa, F. Telomere binding protein Taz1 establishes Swi6 heterochromatin independently of RNAi at telomeres. Curr. Biol. 15, 1808-1819 (2005).

24. Matsuda, A. et al. Highly condensed chromatins are formed adjacent to subtelomeric and decondensed silent chromatin in fission yeast. Nat. Commun. 6, 7753 (2015).

25. Sugawara, N. F. DNA sequences at the telomeres of the fission yeast $S$. pombe. Ph.D. Thesis, Harvard University (1988)

26. Leupold, U. Die vererbung von homothallie und heterothallie bei Schizosaccharomyces pombe. C. R. Trav. Lab. Carlsberg Ser. Physiol. 24, 381-480 (1950).

27. Ohno, Y., Ogiyama, Y., Kubota, Y., Kubo, T. \& Ishii, K. Acentric chromosome ends are prone to fusion with functional chromosome ends 
through a homology-directed rearrangement. Nucleic Acids Res. $\mathbf{4 4}$ 232-244 (2016).

28. Tashiro, S. et al. Shugoshin forms a specialized chromatin domain at subtelomeres that regulates transcription and replication timing. Nat. Commun. 7, 10393 (2016).

29. Nakamura, T. M., Cooper, J. P. \& Cech, T. R. Two modes of survival of fission yeast without telomerase. Science 282, 493-496 (1998).

30. Tusso, S. et al. Ancestral admixture is the main determinant of global biodiversity in fission yeast. Mol. Biol. Evol. 36, 1975-1989 (2019).

31. Bochman, M. L., Paeschke, K. \& Zakian, V. A. DNA secondary structures: stability and function of G-quadruplex structures. Nat. Rev. Genet. 13, 770-780 (2012).

32. Chaudari, A. \& Huberman, J. A. Identification of two telomere-proximal fission yeast DNA replication origins constrained by nearby cis-acting sequences to replicate in late $\mathrm{S}$ phase. F1000Res. 1, 58 (2012).

33. Jeffares, D. C. et al. The genomic and phenotypic diversity of Schizosaccharomyces pombe. Nat. Genet. 47, 235-241 (2015).

34. Fujita, I., Tanaka, M. \& Kanoh, J. Identification of the functional domains of the telomere protein Rap1 in Schizosaccharomyces pombe. PLoS ONE 7, e49151 (2012).

35. Cooper, J. P., Nimmo, E. R., Allshire, R. C. \& Cech, T. R. Regulation of telomere length and function by a Myb-domain protein in fission yeast. Nature 385, 744-747 (1997).

36. Kanoh, J. \& Ishikawa, F. spRap1 and spRif1, recruited to telomeres by Taz1, are essential for telomere function in fission yeast. Curr. Biol. 11, 1624-1630 (2001).

37. Chen, Y. et al. A conserved motif within RAP1 has diversified roles in telomere protection and regulation in different organisms. Nat. Struct. Mol. Biol. 18, 213-221 (2011).

38. Deng, W. et al. Fission yeast telomere-binding protein Taz1 is a functional but not a structural counterpart of human TRF1 and TRF2. Cell Res. 25, 881-884 (2015).

39. Kozak, M. An analysis of 5'-noncoding sequences from 699 vertebrate messenger RNAs. Nucleic Acids Res. 15, 8125-8148 (1987).

40. Young, E., Abid, H. Z., Kwok, P. Y., Riethman, H. \& Xiao, M. Comprehensive analysis of human subtelomeres by whole genome mapping. PLoS Genet. 16, e1008347 (2020).

41. Yue, J. X. et al. Contrasting evolutionary genome dynamics between domesticated and wild yeasts. Nat. Genet. 49, 913-924 (2017).

42. Ferreira, M. G. \& Cooper, J. P. Two modes of DNA double-strand break repair are reciprocally regulated through the fission yeast cell cycle. Genes Dev. 18, 2249-2254 (2004).

43. Miller, K. M., Rog, O. \& Cooper, J. P. Semi-conservative DNA replication through telomeres requires Tazl. Nature 440, 824-828 (2006).

44. Takikawa, M., Tarumoto, Y. \& Ishikawa, F. Fission yeast Stn1 is crucial for semi-conservative replication at telomeres and subtelomeres. Nucleic Acids Res. 45, 1255-1269 (2017)

45. Ivanova, T. et al. Budding yeast complete DNA synthesis after chromosome segregation begins. Nat. Commun. 11, 2267 (2020)

46. Kramara, J., Osia, B. \& Malkova, A. Break-induced replication: the where, the why, and the how. Trends Genet. 34, 518-531 (2018).

47. Smith, C. E., Llorente, B. \& Symington, L. S. Template switching during breakinduced replication. Nature 447, 102-105 (2007).

48. Deem, A. et al. Break-induced replication is highly inaccurate. PLoS Biol. 9, e1000594 (2011).

49. Pardo, B. \& Aguilera, A. Complex chromosomal rearrangements mediated by break-induced replication involve structure-selective endonucleases. PLoS Genet. 8, e1002979 (2012).

50. Anand, R. P. et al. Chromosome rearrangements via template switching between diverged repeated sequences. Genes Dev. 28, 2394-2406 (2014).

51. Sakofsky, C. J. et al. Break-induced replication is a source of mutation clusters underlying kataegis. Cell Rep. 7, 1640-1648 (2014).

52. Sakofsky, C. J. et al. Translesion polymerases drive microhomology-mediated break-induced replication leading to complex chromosomal rearrangements. Mol. Cell 60, 860-872 (2015).

53. Wang, X. \& Baumann, P. Chromosome fusions following telomere loss are mediated by single-strand annealing. Mol. Cell 31, 463-473 (2008).

54. Behm-Ansmant, I. et al. mRNA quality control: an ancient machinery recognizes and degrades mRNAs with nonsense codons. FEBS Lett. 581, 2845-2853 (2007).

55. Mandell, J. G., Goodrich, K. J., Bahler, J. \& Cech, T. R. Expression of a RecQ helicase homolog affects progression through crisis in fission yeast lacking telomerase. J. Biol. Chem. 280, 5249-5257 (2005).

56. Fantes, P. A. \& Hoffman, C. S. A Brief history of Schizosaccharomyces pombe research: a perspective over the past 70 years. Genetics 203, 621-629 (2016).

57. Bahler, J. et al. Heterologous modules for efficient and versatile PCR-based gene targeting in Schizosaccharomyces pombe. Yeast 14, 943-951 (1998).
58. Forsburg, S. L. \& Rhind, N. Basic methods for fission yeast. Yeast 23, 173-183 (2006).

59. Moreno, S., Klar, A. \& Nurse, P. Molecular genetic analysis of fission yeast Schizosaccharomyces pombe. Methods Enzymol. 194, 795-823 (1991).

60. Chen, S., Zhou, Y., Chen, Y. \& Gu, J. fastp: an ultra-fast all-in-one FASTQ preprocessor. Bioinformatics 34, i884-i890 (2018).

61. Koren, S. et al. Canu: scalable and accurate long-read assembly via adaptive kmer weighting and repeat separation. Genome Res. 27, 722-736 (2017).

62. Marcais, G. et al. MUMmer4: A fast and versatile genome alignment system. PLoS Comput. Biol. 14, e1005944 (2018).

63. Larsson, A. AliView: a fast and lightweight alignment viewer and editor for large datasets. Bioinformatics 30, 3276-3278 (2014).

64. Katoh, K., Misawa, K., Kuma, K. \& Miyata, T. MAFFT: a novel method for rapid multiple sequence alignment based on fast Fourier transform. Nucleic Acids Res. 30, 3059-3066 (2002)

65. Li, H. Minimap2: pairwise alignment for nucleotide sequences. Bioinformatics 34, 3094-3100 (2018).

66. Vaser, R., Sovic, I., Nagarajan, N. \& Sikic, M. Fast and accurate de novo genome assembly from long uncorrected reads. Genome Res. 27, 737-746 (2017).

67. Walker, B. J. et al. Pilon: an integrated tool for comprehensive microbial variant detection and genome assembly improvement. PLOS ONE 9, e112963 (2014).

\section{Acknowledgements}

We thank H. Kohno for valuable advices on analyses of NGS data, S. Kuraku, H. Innan H. Michida, T. Nakagawa, and H. Niki for insightful comments, H. Maekawa for critical reading of the manuscript, C. Hoffman, C. Shimoda, M. Yamamoto, and T. Nakamura for lectures on the history of $S$. pombe strains, and all of the lab members for discussion and support. Computations were partially performed on the NIG supercomputer at ROIS National Institute of Genetics. This work was supported by Japan Society for the promotion of Science (JSPS) KAKENHI (JP17H03606, JP19H05262, JP20H03185, and JP20H05388), Ohsumi Frontier Science Foundation, and the publication support from Initiative for the Implementation of the Diversity Research Environment of Osaka University to J.K., and Grant-in-Aid for JSPS Research Fellows to Y.O.

\section{Author contributions}

J.K. conceived the project, Y.O. and J.K. analyzed sequence data, T.K., S.T., and J.K. designed and performed experiments. Y.T. and Y.D. contributed to the analysis of $S H$ regions. Y.O. and J.K. analyzed data and wrote the manuscript.

\section{Competing interests}

The authors declare no competing interests.

\section{Additional information}

Supplementary information is available for this paper at https://doi.org/10.1038/s41467020-20595-1.

Correspondence and requests for materials should be addressed to J.K.

Peer review information Nature Communications thanks Sigurd Braun and Maria Teresa Teixeira for their contribution to the peer review of this work. Peer reviewer reports are available.

\section{Reprints and permission information is available at http://www.nature.com/reprints}

Publisher's note Springer Nature remains neutral with regard to jurisdictional claims in published maps and institutional affiliations.

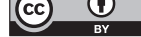

Open Access This article is licensed under a Creative Commons Attribution 4.0 International License, which permits use, sharing, adaptation, distribution and reproduction in any medium or format, as long as you give appropriate credit to the original author(s) and the source, provide a link to the Creative Commons license, and indicate if changes were made. The images or other third party material in this article are included in the article's Creative Commons license, unless indicated otherwise in a credit line to the material. If material is not included in the article's Creative Commons license and your intended use is not permitted by statutory regulation or exceeds the permitted use, you will need to obtain permission directly from the copyright holder. To view a copy of this license, visit http://creativecommons.org/ licenses/by/4.0\%

(C) The Author(s) 2021 